\title{
DNA-protein interaction studies: a historical and comparative analysis
}

\author{
Ricardo André Campos Ferraz ${ }^{1,2}$, Ana Lúcia Gonçalves Lopes 1,2, Jessy Ariana Faria da Silva²,3, \\ Diana Filipa Viana Moreira ${ }^{1,2}$, Maria João Nogueira Ferreira ${ }^{1,2}$ and Sílvia Vieira de Almeida Coimbra ${ }^{1,2^{*}}$ (D)
}

\begin{abstract}
DNA-protein interactions are essential for several molecular and cellular mechanisms, such as transcription, transcriptional regulation, DNA modifications, among others. For many decades scientists tried to unravel how DNA links to proteins, forming complex and vital interactions. However, the high number of techniques developed for the study of these interactions made the choice of the appropriate technique a difficult task. This review intends to provide a historical context and compile the methods that describe DNA-protein interactions according to the purpose of each approach, summarise the respective advantages and disadvantages and give some examples of recent uses for each technique. The final aim of this work is to help in deciding which technique to perform according to the objectives and capacities of each research team. Considering the DNA-binding proteins characterisation, filter binding assay and EMSA are easy in vitro methods that rapidly identify nucleic acid-protein binding interactions. To find DNA-binding sites, DNA-footprinting is indeed an easier, faster and reliable approach, however, techniques involving base analogues and base-site selection are more precise. Concerning binding kinetics and affinities, filter binding assay and EMSA are useful and easy methods, although SPR and spectroscopy techniques are more sensitive. Finally, relatively to genome-wide studies, ChIP-seq is the desired method, given the coverage and resolution of the technique. In conclusion, although some experiments are easier and faster than others, when designing a DNA-protein interaction study several concerns should be taken and different techniques may need to be considered, since different methods confer different precisions and accuracies.
\end{abstract}

Keywords: ChIP, DNA-footprinting, DNA-protein interaction, EMSA, SELEX, SPR

\section{Introduction}

It has been known since the second half of the last century that the binding of a protein to a DNA molecule has a very important role in the function of a living cell and in life's sustainability itself. For many decades and representing a big segment of the molecular biology research conducted, scientists tried to unravel how DNA links to proteins, forming complex and vital interactions. In the early beginning of these studies, even before the

*Correspondence: scoimbra@fc.up.pt

${ }^{1}$ Departamento de Biologia, Faculdade de Ciências da Universidade do Porto, Porto, Portugal

Full list of author information is available at the end of the article publication of the DNA molecular structure, Stedman and Stedman [1] already referred to histones as potential regulators of the DNA biological activity. Since then, scientists have not abandoned this research field, having unravelled many details about the crucial interaction between proteins and DNA. This interaction is responsible for essential molecular and cellular mechanisms, such as transcription, transcriptional regulation, recombination, replication, DNA repair, viral infection, DNA packing and DNA modifications [2]. The studies usually performed were either from a purely chemical perspective, analysing the structure of the complex formed, or from a transcriptomic level, investigating if a certain protein does bind to a particular DNA or gene and the 
interference of this interaction in gene expression, existing great intersection between both approaches [3].

From a molecular point of view, lifting the veil from the way a DNA molecule binds to a protein and starting to distinguish some patterns and possible favoured interactions between amino acids and DNA base sequences, in the sixties Leng and Felsenfeld [4] discovered that polylysine polypeptides interact preferably with A-T-rich DNA, while polyarginine connects desirably to G-C-rich DNA. A decade later, Seeman et al. [5] shed a little more light upon the structure of these interactions and, using the hydrogen-bonding atoms identified by these researchers on DNA base edges, suggested that specific amino acid side chains recognise certain nucleotides and that there is an increased likelihood that these interactions are more specific in the DNA major groove than in the minor groove. Indeed, a few years later, the model building studies that resulted from the McKay and Steitz [6] pioneer detection of a DNA-protein complex using $\mathrm{X}$-ray crystallography suggested that Escherichia coli catabolite gene activator protein (CAP) does bind to the DNA major groove. Later, Pabo and Sauer [7] and Matthews [8] continued to study the amino acid-base connection and included electrostatic and van der Waals interactions in the models developed. Nowadays, despite knowing that there are some preferred interactions, it is accepted that protein families bind to DNA in different ways depending on the complex formed, not existing a straightforward correspondence between amino acids and bases of nucleic acids [9]. The majority of solely chemical investigations related to DNA-protein binding performed more recently focus on a specific interaction and the techniques used in this context enable to collect data related to several aspects such as the protein's size, the DNA-binding site, the strength of the binding, the effects of the protein binding on the structure of the DNA and individual groups or specific bases involved in the interaction [3].

As for the study of transcription factors (TFs) and the regulation of gene expression, it was also in the sixties that François Jacob and Jacques Monod discovered the genetic regulatory mechanism in prokaryotes moderated by the lac operon [10]. Many findings followed that managed to decipher the mechanism that controls gene expression. Generally, the studies that use these techniques are centred on a specific protein, somehow related to the phenotype being investigated, which is suspected of binding to the promoter region of a potential target gene. The aim of each technique may be to recognise DNA-binding proteins in a cell extract, identify the DNA-binding site, analyse the specificity of the binding, or simply confirm if a given protein does bind to the respective alleged target genes and determine the effects of the binding in these genes' expression, envisioning to reveal novel insights into gene regulatory systems [3].

This review intends to compile and briefly describe the majority of the existing techniques, and the respective variants, that enable to access information related to DNA-protein interactions, trying to combine them according to their purpose, knowing that there is an overlap between certain methods. The positive and negative aspects, as well as some improvements and slight modifications performed to each procedure referred in recent studies will also be noted. The final aim of this article is to aid researchers in the moment of deciding which technique to perform according to the objectives and capacities of each research team, hoping to contribute to the increase of the current knowledge related to DNA-protein interactions and gene regulatory networks.

\section{Studying DNA-protein interactions DNA-binding proteins characterisation Filter binding assay and electrophoretic mobility shift assay (EMSA)}

The interaction between nucleic acids and proteins was not yet totally described when Yarus and Berg [11] developed the filter binding assay that relies in the fact that a major part of proteins can be retained in a nitrocellulose membrane. In case the protein under study does bind to a nucleic acid, subsequently the complex may also be held in the nitrocellulose filter. This method is quite inexpensive, simple and relatively rapid, starts by the extraction and purification of the protein of interest and radio-labelling of the nucleic acid, followed by the binding reaction and ends in the filtering technique, which consists in applying vacuum to a porous plastic disc placed bellow a nitrocellulose filter that is impregnated with a solution containing the binding reaction. Then, the results are revealed and the quantities of bound nucleic acid are calculated using a phosphorimager [12] (Fig. 1).

Membrane filters were already being used for some time to detect binding between molecules [13], when Jones and Berg [14] tested these membranes to study the interaction between DNA and proteins. However, this last experiment was a bit dubious since the protein itself would not bind to the membrane. It was around this time that Yarus and Berg [11] introduced the nitrocellulose membranes to verify the recognition of a tRNA by an enzyme and, just a year later, Riggs et al. [15] continued improving this method by expanding its applicability, managing to properly detect the binding of a protein to DNA, more specifically the lac repressor-operator interaction. This new approach replaced the tedious and limited techniques that were being used until then, such as glycerol or sucrose density gradients and DNA columns. 


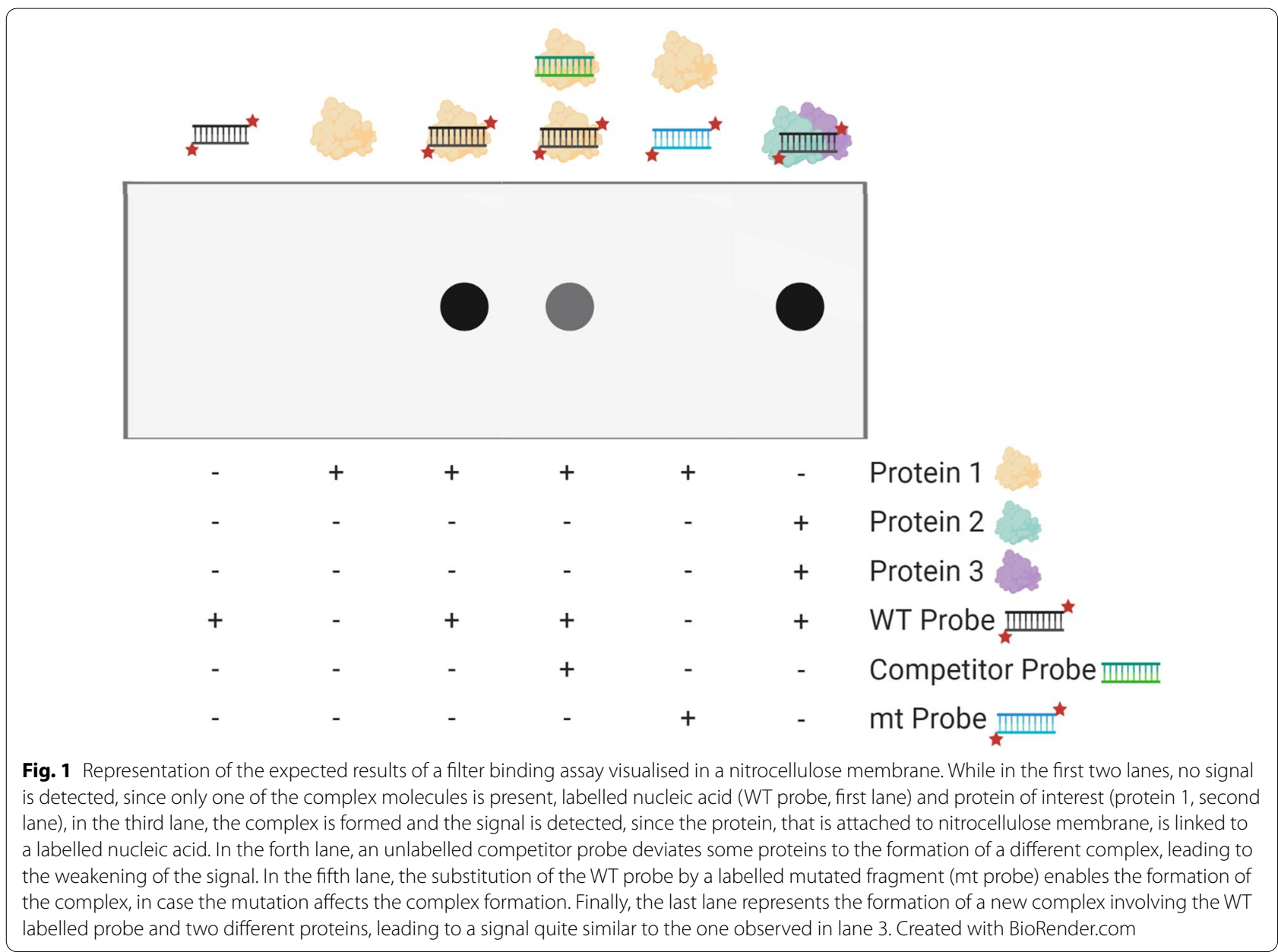

However, this procedure presents several drawbacks that have led to its disuse. Firstly, not all the proteins bind to nitrocellulose membranes and some even denature when clinging to these filters. Moreover, if the interaction between the molecules of the complex is not strong enough, it may not withstand the filtration process [16]. Furthermore, using this method one cannot recover and analyse the composition of the binding reaction resulting products, making it impossible to determine if a DNA molecule binds to more than one protein, since the DNA only needs to interact to a single protein in order to be retained in the filter [17] (Fig. 1). Additionally, if singlestranded nucleic acids adhere randomly to the filter, which happens under certain solution conditions, it may result in undesirable interferences that might obscure the true binding signal [18].

Finally, a technique was developed that uses gel electrophoresis and that surpasses these inconveniences. Fried and Crothers [17] and Garner and Revzin [19] created the electrophoretic mobility shift assay (EMSA), which consists in evaluating if a protein causes a retardation in the electrophoretic run of a nucleic acid fragment when bound to it in a complex compared to the run of the same nucleic acid not bound to a protein. Like the filter binding assay mentioned above, EMSA tests the nucleic acid-protein interaction qualitatively, but unlike the first one, EMSA also does it quantitatively, since the mobility of the nucleic acid fragment in the gel decreases as the number of proteins bound to it increases. So, this method assesses not only the stoichiometric ratio of protein linked to the nucleic acid, but also the relative binding affinities of a certain protein for two different nucleic acids [17]. Furthermore, if, in the beginning of the electrophoresis, the nucleic acid fragments that are not involved in any interaction enter the electrophoretic gel before the dissociation of the complexes formed, it is also possible to quantify the concentration of unbound nucleic acid fragments and, thus, the concentration of the complexes in the binding reaction [19].

Usually, the technique starts by mixing the protein, present in a crude cell extract or purified, with the labelled nucleic acid and an appropriate buffer, under 
the right specific conditions and concentrations for the binding reaction to occur, and the final products are separated in a non-denaturing gel electrophoresis. To obtain purified protein, one can insert the coding sequence of the gene that codifies the protein of interest in an expression vector for bacteria or yeast transformation, induce the transcription and translation of this gene and extract and purify the protein using purification columns. As for the DNA fragments, these are usually labelled with radioisotopes, covalent or noncovalent fluorophores or biotin. The results are then observed by autoradiography, fluorescence imaging, chemiluminescent imaging and/or chromophore deposition (Fig. 2). It must be noted that certain parameters must be adjusted so that all this process succeeds, most of which were tested and resumed by Fried [20] and Hellman and Fried [18].

Such as filter binding assays $[12,16]$, the EMSA protocol can also include the use of a competing unlabelled nucleic acid, which is especially useful when dealing with crude extracts [3], where non-specific complexes are created by secondary binding activities. The incorporation of this additional reagent enables the discrimination of specific and non-specific unwanted bindings when the protein under study binds to the target nucleic acid with a higher affinity than it links to the competitor and when the other secondary binding activities do not differentiate between target and competitor sequences. Still, the competitor nucleic acid may also bind to the protein of interest, which will result in a decrease of the specific binding

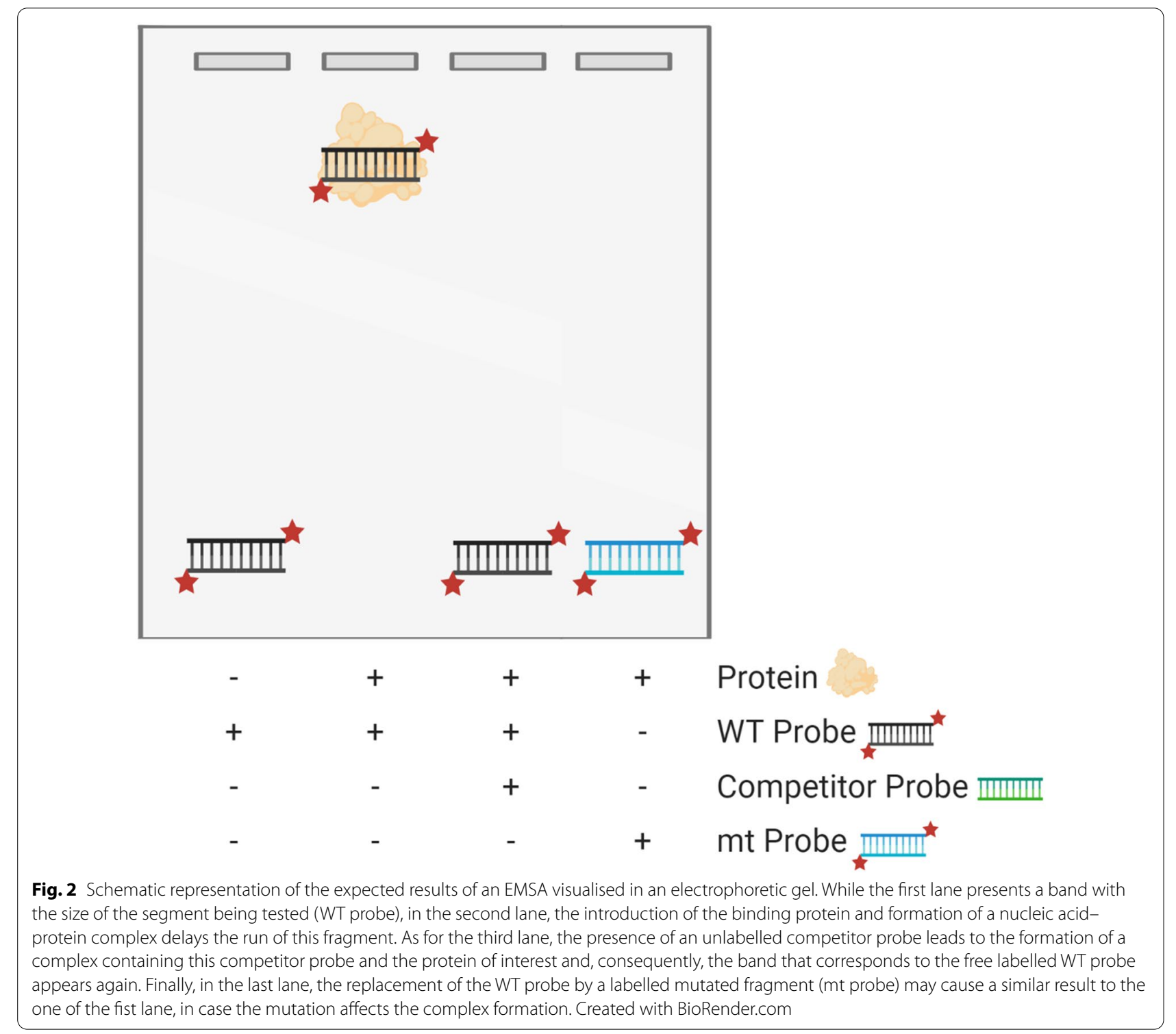


(Figs. 1, 2). In order to avoid this possibility, one should experiment several competitor concentrations with the purpose of improving the distinction between specific and non-specific interactions. Usually, poly $\mathrm{d}(\mathrm{A}-\mathrm{T})$, poly $\mathrm{d}(\mathrm{I}-\mathrm{C})$ and genomic DNAs are used as competitors for DNA-protein bindings [18], while tRNA is preferred when dealing with RNA-protein interactions [16].

Overall, EMSA is easy to execute, but powerful and sensitive, comprising a broad spectrum of binding conditions and enabling the use of small concentrations of protein and nucleic acid and small sample volumes, especially when the nucleic acid is radio-labelled. However, fluorescence, chemiluminescence and immunohistochemical detection can also be used in case a high sensitivity is not necessary. Concerning the advantages related to the nucleic acid used, this assay is able to test oligonucleotides with a large variety of sizes, which can go from short nucleic acids to molecules with thousands of nucleotides or base pairs, and structures, which can be single, double-stranded, triplex or quadruplex nucleic acids, or even small circular DNAs. As for the protein under study, proteins used in EMSA can have very distinct sizes and still efficiently provide mobility shifts. Using ESMA, one can even distinguish how proteins are spread across different interactions with various nucleic acid molecules in one solution, as well as the existence of complexes with different protein stoichiometry and/or binding site distribution [18].

Nevertheless, EMSA also presents some disadvantages. One of them is that, during the electrophoresis run, partial or total dissociation of the nucleic acid-protein complex can happen, or, on the contrary, many complexes acquire more stability in the gel than in a free solution. Either way, the results can be misleading, not reflecting the state of the interaction before the loading of the sample in the electrophoresis gel. To solve this issue, shorter electrophoresis times are suggested. Another drawback is that, in case a shift is observed in the mobility of a nucleic acid during the electrophoresis step, it is not necessarily due to the size of the proteins that are bound to it. Many other factors may be in the origin of the mobility shift, such as the conformation and structure of the complex. Moreover, this assay does not give information about the location of the sequences of the nucleic acid that are linked to the protein. These last two problems can be addressed using alternative techniques that complement or replace EMSA and that are referred in the next sections [18].

Overall, filter binding assay and EMSA are two important techniques that can help in identifying nucleic acidprotein interactions quite easily and rapidly. Advantages, disadvantages and applications of these two methods are resumed in Table 1. Other techniques may cooperate in order to obtain more information about this interaction. Some of them are examined in the following sections.

\section{Cross-linking}

An EMSA technique drawback is the dependence of its results on several factors besides the protein size. This assay does not allow to identify nor reveal the molecular weights of the proteins present in the complex being studied. Obtaining this information is crucial especially when dealing with impurified or partially purified cell extracts, since they contain many nucleic acid-binding proteins, being that different proteins can bind to the nucleic acid of interest. Thus, other techniques need to be performed complementary to EMSA to clarify which protein is causing the electrophoretic mobility shift [18].

In the seventies, quaternary structures related to interactions between proteins were analysed using cross-linking techniques [21]. Covalent cross-links were produced between proteins belonging to the same complex, so that they could be extracted and studied in subsequent steps. Other approaches also developed, enabled the generation of cross-links between DNA and proteins from the same complex [22]. Generally, each distinct procedure starts by forming or isolating the DNA-protein complexes, excluding free probes and nonspecific complexes. A cross-linker agent is then applied and the specific complex is removed and precipitated, being finally analysed [3] (Fig. 3a). In some of the following approaches, the cross-linker may be applied prior to the formation of the complex to increase the affinity of the DNA to bind to proteins.

There are different cross-linker agents and strategies, which can be grouped into chemical and photo-crosslinking. Chemical cross-linking techniques often use formaldehyde and glutaraldehyde as the cross-linker agents. These two agents are usually employed in the study of nucleoproteins, such as histones [22]. Brutlag et al. [23] found that the presence of DNA in formaldehyde treatment of nucleohistones generates DNA-protein complexes, due to the methylene bridges formed between adjacent amino groups. It was also reported by the same researchers that the increase of the formaldehyde concentration results in a higher amount of bounded protein. However, they concluded that high concentrations of formaldehyde causes secondary alterations in the histones structure or composition, which can be a disadvantage of this cross-linker agent [23]. As for glutaraldehyde, while Chalkley and Hunter [24] stated that, unlike formaldehyde, glutaraldehyde creates bonds primarily between histones, without excluding a few DNA-histone links, Kuykendall and Bogdanffy [25] reported that glutaraldehyde is a very potent cross-linker, almost as effective as formaldehyde, most probably because of its difunctional 


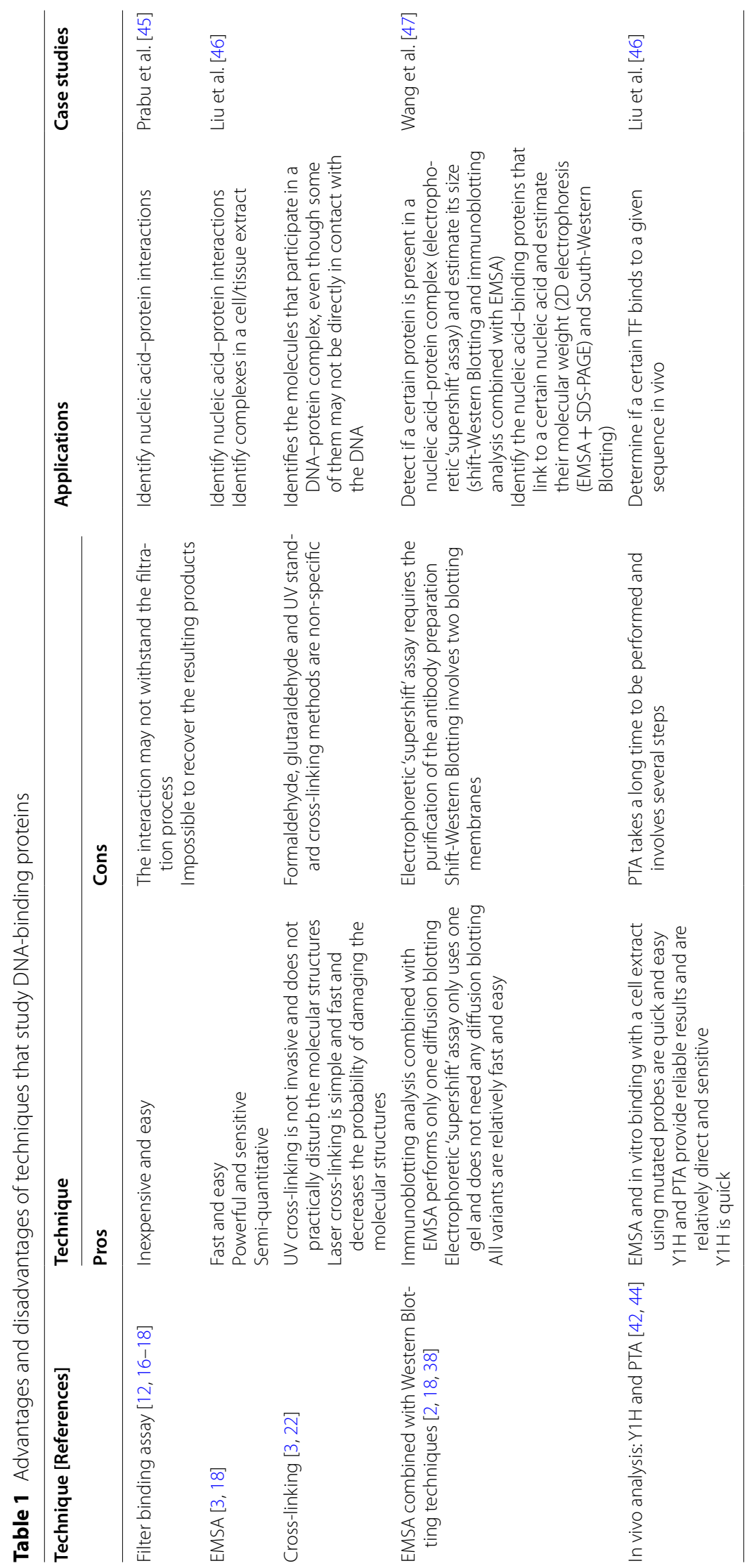



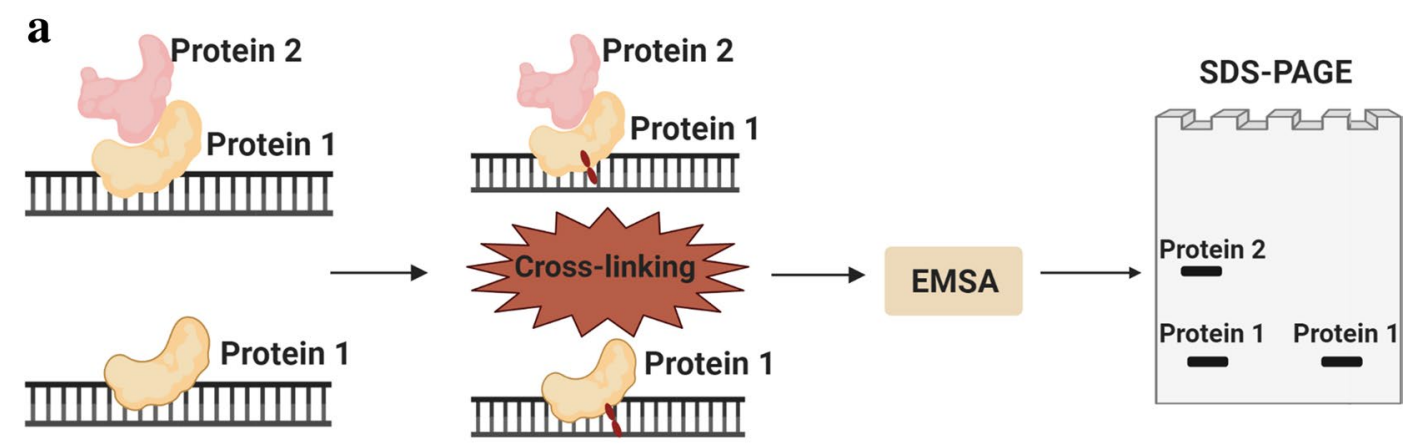

b

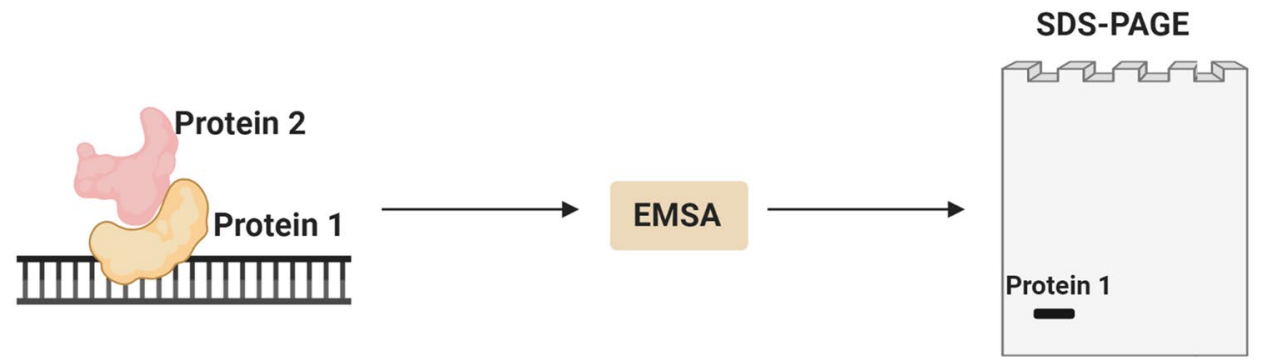

Fig. 3 Comparison between the cross-linking procedure (a) and South-Western Blotting (b). In the cross-linking tehcnique (a) a nucleic acid is cross-linked to the respective binding proteins, ensuring that all proteins present in the complex are detected in a SDS-PAGE performed after an EMSA (that isolates the complex under study), independently of being in direct contact with the nucleic acid (Protein 1) or not (Protein 2). As for South-Western Blotting (b), after an EMSA, the complex is subjected to a SDS-PAGE without being exposed to a cross-linker and, consequently, only the proteins in direct contact with the nucleic acid will be detected. Created with BioRender.com

nature. Chemical cross-linking can also be applied when studying virus structures, as it was performed by Chattoraj and Inman [26], that used formaldehyde as a crosslinker agent when trying to unravel the arrangement of DNA in bacteriophage heads, and Thomas et al. [27], who employed 1-ethyl-3(3-dimethyl-aminopropyl) carbodiimide with a similar purpose.

Photo-cross-linking was firstly performed by Smith [28], that irradiated DNA mixed with protein with a UV light of $254 \mathrm{~nm}$, near the absorbance maximum for DNA and some amino acid side chains, using a mineral lamp. This causes cross-link of the DNA to several distinct amino acid side chains, being a non-specific method. Among other works, this method was applied in studying cross-linking between histones and DNA and virus replication $[29,30]$. This technique's advantage lies in the fact that this procedure is not invasive and does not practically disturb the structures of the molecules under study [22]. Nevertheless, the method was improved. Two decades after the first cross-linking experiment using UV light, Harrison et al. [31] inflicted a very short UV pulse from a laser on a complex formed by E. coli RNA polymerase and T7 DNA, inducing the cross-link between these two molecules. Apart from being a simple and fast method, this upgrade to the UV cross-linking technique decreases even more the chance of damaging the structure of the molecules being analysed, since it uses short and powerful pulses. Besides, this approach can also be applied in in vivo studies, without affecting the metabolism of a cell, as it happens with conventional UV cross-linking. It also enables the immediate cross-link of a complex involving several proteins. However, this procedure may cause more chain scission in DNA than the standard UV cross-linking method referred before [31].

Overall, cross-linking techniques have the advantage of identifying the molecules that participate in a DNA-protein complex, even though some of them may not be directly in contact with the DNA. Nevertheless, when analysing the molecular weight of the proteins present in the complex through a sodium dodecyl sulphate-polyacrylamide gel electrophoresis (SDSPAGE) (Fig. 3a), DNase I might be necessary in order to remove the DNA attached to the proteins, risking a decrease of the signal, since the presence of DNA may lead to an irregular run, giving an incorrect estimation of the molecular weight [3]. Advantages, disadvantages and applications of some cross-link variants are resumed in Table 1. 


\section{EMSA combined with western blotting techniques}

Western Blotting was initially developed by Towbin et al. [32] enabling the identification of proteins using specific antibodies, after performing an electrophoresis. It can be used to detect specific proteins present in a cell extract. Several techniques were developed that combine EMSA with Western Blotting, making it possible to detect a specific DNA-protein interaction, as well as the size of the protein, within a cell extract.

Kristie and Roizman [33] created an approach called electrophoretic 'supershift' assay, which consists in adding to the nucleic acid-protein mixture an antibody against a candidate protein. In case the protein causing the mobility shift of the nucleic acid is the one of interest, the presence of the antibody will cause a secondary mobility shift, or the blocking of the complex formation. As for the moment when the antibody is added, the results obtained with this method will be different if the antibody is included before or after the formation of the nucleic acid-protein complex, mainly if the target protein contains epitopes in the DNA-binding surface. Moreover, the antibody preparation needs to be as pure as possible, since contaminants may interfere in the stability or mobility of the nucleic acid-protein complexes. Alternatively, a control antibody reaction can also be prepared to detect possible interferences of the antibody preparation $[18,34]$.

Singh et al. [35] also created a technique fusing EMSA and Western Blotting, the South-Western Blotting, according to which the complex identification uses radioactively labelled oligonucleotides. After separating the cell proteins by SDS-PAGE and transferring to nitrocellulose filters, these are either incubated with an oligonucleotide corresponding to the nucleic acid of interest, or with a point-mutated variation of the same oligonucleotide that does not form the specific complex, serving as control. An EMSA is performed previously to verify the adequacy of the control oligonucleotide designed. The filters are then washed and exposed for autoradiography (Fig. 3b). The differences between the results obtained with each oligonucleotide represent the specific nucleic acid-protein complexes. If more than one specific band is observed, it means that the nucleic acid sequence under study is recognised by a family of proteins. Although providing precise information about the molecular weight of a nucleic acid-binding protein, this procedure only succeeds if the protein is in direct contact with the nucleicacid and if the nucleic acid binding does not involve an heteromeric protein complex [3].

Demczuk et al. [36] performed a method involving simultaneous immunoblotting analysis with EMSA, known as 'shift-Western Blotting', according to which a nitrocellulose filter and an anion-exchange membrane are piled following native gel electrophoresis to identify the nucleic acid-protein complex by autoradiography of the DNA blot, present in the anion-exchange membrane, and by immunoblotting of the protein blot, obtained in the nitrocellulose filter. However, Granger-Schnarr et al. [37] and Chen and Chang [38] created a combined analysis that only performs one simple diffusion blotting into a polyvinylidene difluoride (PVDF) membrane after electrophoresis, being the gel directly analysed by autoradiography and the PVDF incubated with a specific antibody and analysed by immunoblotting. This last procedure is especially powerful, since it enables the identification of each component of a multiple DNA-TF complex, replicating the immunoblotting analyses using antibodies specific for each TF. Finally, in 2006, Stead et al. [39] implemented a two-dimensional gel electrophoresis, that combined EMSA, SDS-PAGE and mass spectrometry in the same gel. After performing an EMSA in a tube gel, this tube is placed above an SDS gel in order to separate the proteins present in the specific complexes obtained in the EMSA according to their molecular weight, which are then identified performing a peptide mass fingerprinting. This method enables the detection of nucleic acid-protein complexes in a cell extract within 4 days, significantly decreasing the need for protein purification and facilitating the identification of all the proteins present in that extract that interact with the nucleic acid being studied. Advantages, disadvantages and applications of some techniques that combine EMSA with Western Blotting are resumed in Table 1.

\section{In vivo analysis: $Y 1 H$ and PTA}

In order to relate binding sites with the TF binding in vivo, systems like the yeast one-hybrid $(\mathrm{Y} 1 \mathrm{H})$ were created. This system, developed by $\mathrm{Li}$ and Herskowitz [40], implicates a vector expressing the TF under study fused to a yeast transcription activation domain, usually GAL4, and a construct containing the gene of interest, or a region of its promoter, more specifically, its cis elements, upstream to the GAL4 promoter and a reporter gene, usually the lac $Z$ gene. These two elements are inserted into a yeast cell and, in case the TF does bind to the gene of interest, the activation domain binds to the promoter and leads to the expression of the reporter gene, being this activation independent of the TF action, either it is an activator or a repressor. Unlike in vitro techniques, this technique employs a system where a cell is involved, providing much more reliable results since it recreates an environment similar to the one where the complex under study is formed [41]. It can be concluded that $\mathrm{Y} 1 \mathrm{H}$ is a relatively direct, quick and sensitive method to determine if a certain TF binds to a given sequence in vivo [42]. 
Still, a yeast cell is quite different from other cells, such as a plant cell. This method does not totally recreate the environment of a plant cell, disregarding some factors that might interfere and determine the DNA-protein interaction being studied. Therefore, in this case, an in planta was developed. The creation of a Transient Expression in Arabidopsis Mesophyll Protoplast (TEAMP) method by Yoo et al. [43], a protoplast transactivation assay (PTA), enabled to perform an experiment similar to $\mathrm{Y} 1 \mathrm{H}$, but using plant cells, more specifically protoplasts, eliminating the risk of drawing false conclusions from techniques that use different organisms, like mentioned above. Ueda et al. [44] used this protocol to study the interaction between the Wuschel related homeobox 8 (WOX8) gene and the WRK2 TF. After identifying the WOX8 cis elements, the group followed the TEAMP protocol and transformed Arabidopsis mesophyll protoplasts, previously transformed with another vector containing the WRKY2 gene linked to the GAL4 activation domain, with the constructs containing the cis elements linked to the GAL4 promoter and a reporter gene. As these protoplasts exhibited reporter gene expression, the group suggested that WRKY2 does bind to the WOX8 gene, which, in this experience, led to the expression of the reporter gene (Fig. 4). Despite being a very complex procedure that involves numerous steps, PTA is a reliable in planta technique when trying to decipher gene expression networks and study gene expression direct regulation. Advantages, disadvantages and applications of the techniques referred are resumed in Table 1.

\section{DNA-binding site localisation Footprinting}

A method usually performed in order to discover the precise sequence of the nucleic acid that binds to a protein is the footprinting. There are several footprinting techniques, each one with a different way of testing if a modification in a particular segment of the nucleic acid interferes or is blocked by protein binding, enabling to identify the location of the binding sequence. All the footprinting variations have in common the ${ }^{32} \mathrm{P}$ endlabelling of the nucleic acid strands being tested; the modification and binding of each nucleic acid strand in parallel reactions; the sequencing of each fragment to identify the modification sites; and, if possible, the quantitative analysis of the "free" and "bound" nucleic acid fractions after sequencing. Footprinting techniques can be divided into "protective" and "interference" methods, being each one the reverse of the other. While in the first the protein binds to the nucleic acid before and "protecting" it from modification, in the latter the nucleic acid is altered first and then tested for protein binding [3].

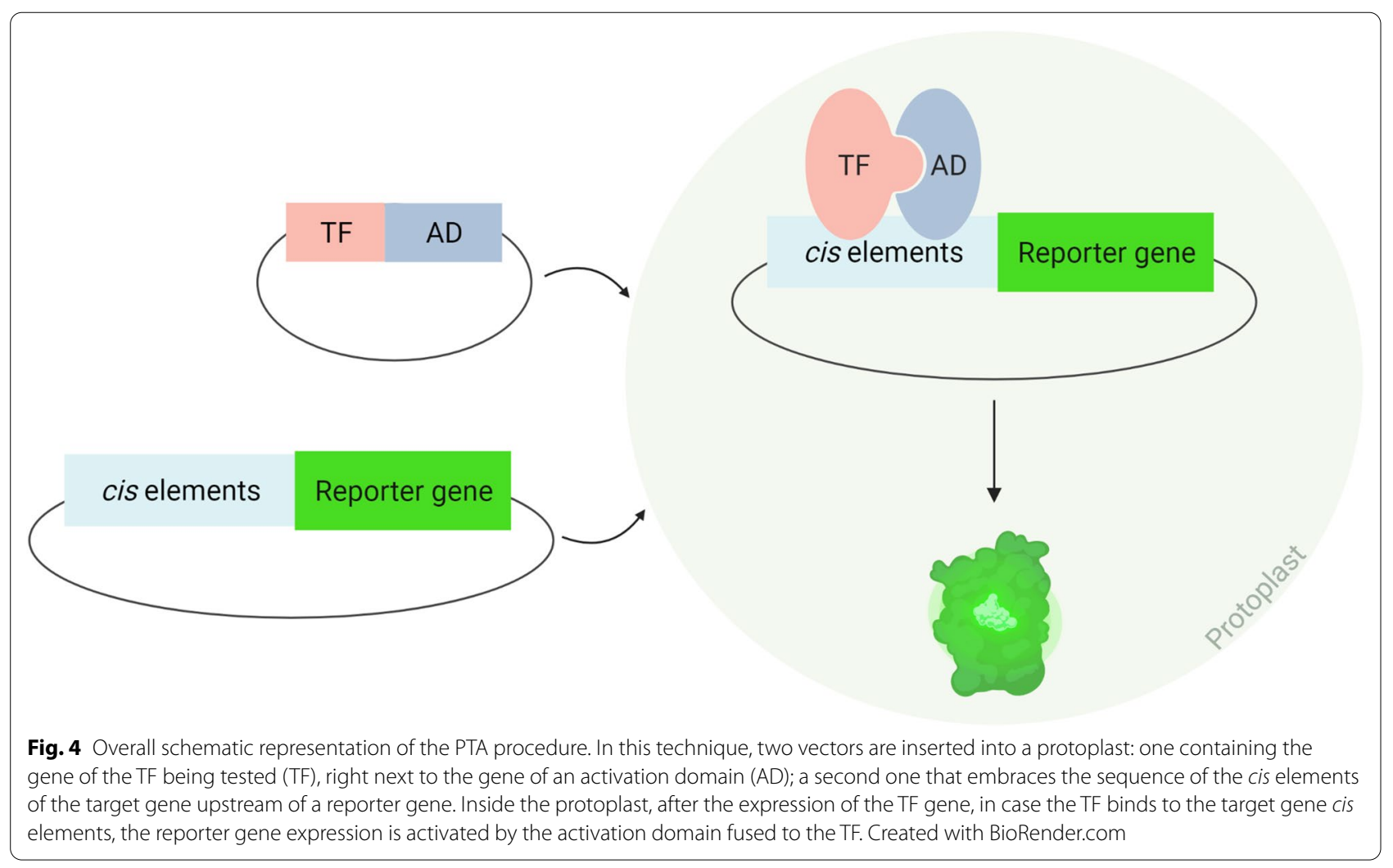


"Protective" footprinting methods, in turn, can be divided into enzymatic or chemical. In both approaches, the nucleic acid of interest is mixed with the protein under study, whether it is present in a crude extract or in a pure sample. Then, the enzymatic or chemical reagent partially cleave the nucleic acid, except for the region protected by the protein, in case it binds to the nucleic acid. Finally, the cleaved nucleic acid is submitted to a denaturing electrophoresis, together with a sequencing ladder, and to autoradiography in order to identify the protected segment, represented by a lacuna in the continuous bands of the restriction products, the footprint, when compared to a track containing the free DNA cleavage products [3] (Fig. 5).

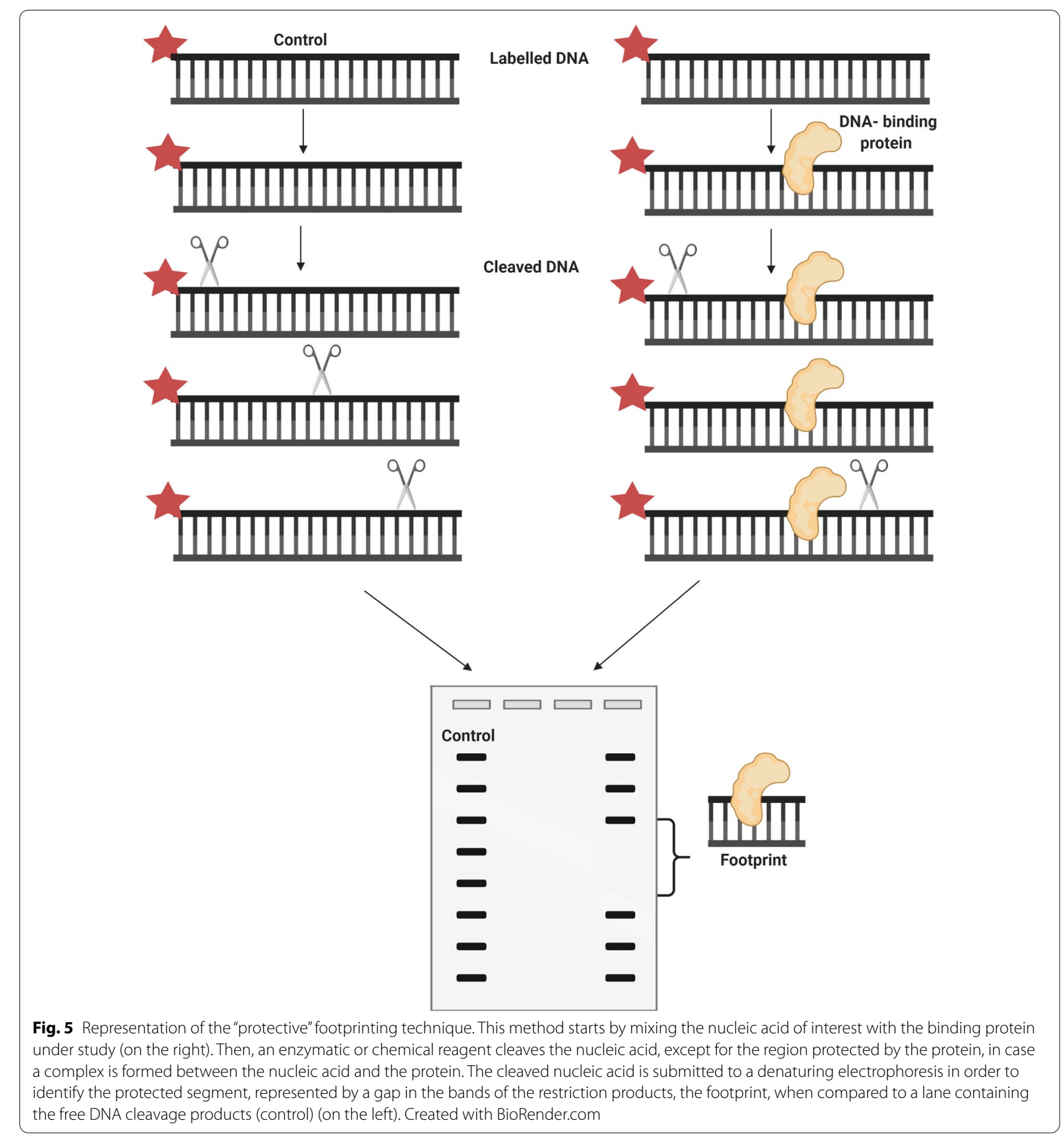


"Protective" footprinting results usually present a background that corresponds to free DNA that was not linked to a protein. This problem can be overcome by adding large amounts of protein, which is not always easy to obtain, especially when dealing with purified samples, and can lead to non-specific binding. Another way to eliminate the background is to perform an EMSA, in order to separate free- and bound-DNA fractions, prior to the footprinting reaction [3]. Papavassiliou and Heumann [48] developed a new method according to which the footprinting reaction is executed in the nondenaturing gel of the EMSA. Thus, the complex is maintained in the gel while being exposed to the footprinting reagents. Moreover, multiple complexes can be separately obtained and subjected to the footprinting agent, enabling to study different binding sites and complexes in the same nucleic acid fragment. Another disadvantage of these in vitro footprinting methods is the disregard for factors existing in cells that may interfere in the complex formation [3]. Nonetheless, Church and Gilbert [49] developed an in vivo footprinting method, starting by treating cells with DMS, since it can penetrate plasma and nuclear membranes. After isolating the genomic DNA of these cells, the altered guanine residues are restricted by piperidine.

Relatively to "interference" footprinting methods, these techniques consist in inflicting modifications on DNA bases and identifying the alterations that inhibit or diminish protein binding. These approaches are more direct and have a higher resolution than the previous ones, since they investigate the impact of each nucleotide on the binding affinity, modifying only one nucleotide per DNA fragment [3].

Overall, footprinting techniques present a low throughput [50] and need optimisation of binding by the protein under study and of the modification reaction of the nucleic acid, being harder to perform than EMSA or filter binding assays. Furthermore, as many nucleic acid fragments are radiolabelled in footprinting methods, the detection of the binding is less sensitive than using EMSA. In addition, given that some proteins bind to nucleic acids in a non-specific way, the results obtained with footprinting procedures may not be so distinct as the ones achieved by EMSA and filter binding assays. Nevertheless, footprinting is the method mostly chosen to identify the sequences of the binding sites. This method can also be applied to study the interactions of a long nucleic acid with several binding proteins, given that big sequences can be resolved on a conventional sequencing gel. Moreover, contrary to EMSA, a footprint signal can be visualised in binding equilibrium conditions [18]. Advantages, disadvantages and applications of the footprinting techniques are resumed in Table 2.

\section{Base analogues}

An expensive and long procedure that can be performed to identify the contribution of each base to the DNAbinding affinity of a protein involves the integration of modified bases at particular sites in the course of the synthesis of both strands that create the DNA duplex [51]. This technique involves assembly and an EMSA test for each specific DNA duplex designed for every alteration being analysed. However, this method provides more accurate results than the footprinting procedures. It enables to access the relative affinity of a protein for DNA fragments that lack particular groups on a base, allowing to understand the contribution of crucial interactions to the binding. This procedure is quite useful to determine the precise bases interfering in the DNA-protein interaction, especially if complementing previous experiments that have pointed towards a specific binding site [3]. The advantages, disadvantages and applications of this method are present in Table 2.

\section{Binding-site selection}

Another radical technique consists in synthesising oligonucleotides that incorporate a random nucleotide mixture at specific positions in a certain sequence. The bases located at determined sites in sequences that bind to the protein under study interaction, usually checked by an EMSA, are established as the preferred bases [3]. In the 90's, new methods were created, that gave a great contribution to the techniques that select binding-sites from random oligonucleotide mixtures. Tuerk and Gold [52] developed the systematic evolution of ligands by exponential enrichment (SELEX) procedure, an in vitro technique that rapidly accesses the oligonucleotides that have an adequate binding affinity to a certain molecular target from a library of random generated oligonucleotides. Basically, this method starts by creating a library of oligonucleotides, followed by incubation with the target protein. Subsequently, the bound and free fractions are separated and the bound oligonucleotides are amplified by PCR, regarding DNA, or by RT-PCR succeeded by in vitro transcription, concerning RNA. This threestrep procedure, target binding, selection and amplification, is called a SELEX round and is repeated numerous times, being some oligonucleotides selected in the last round, called aptamers, sequenced (Fig. 6). In previous techniques, random nucleotides were cloned into plasmids that were then used in transformation procedures and individual transformants were selected according to their phenotypes. Thus, the SELEX method presents several advantages over the procedures performed until its creation. Firstly, it does not depend on cloning to select the oligonucleotides. Furthermore, it does not require the analysis of phenotypes, which may depend on various 


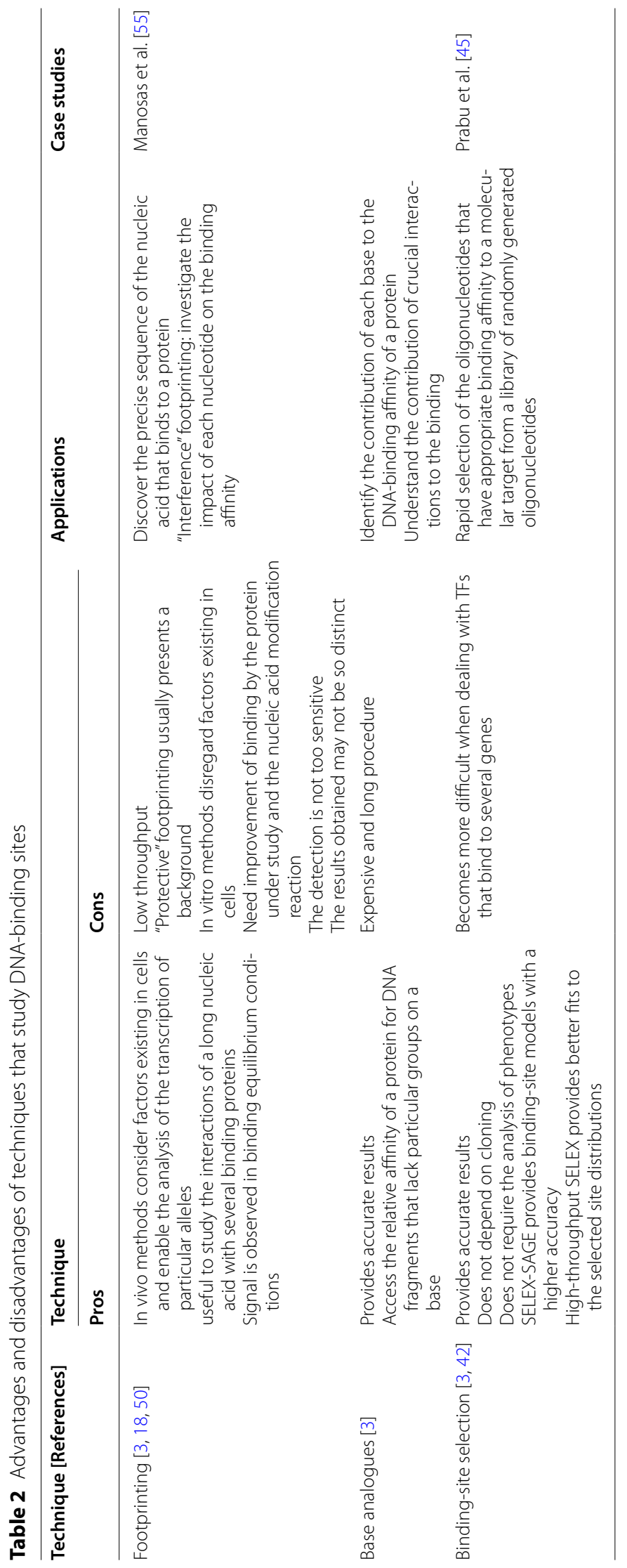




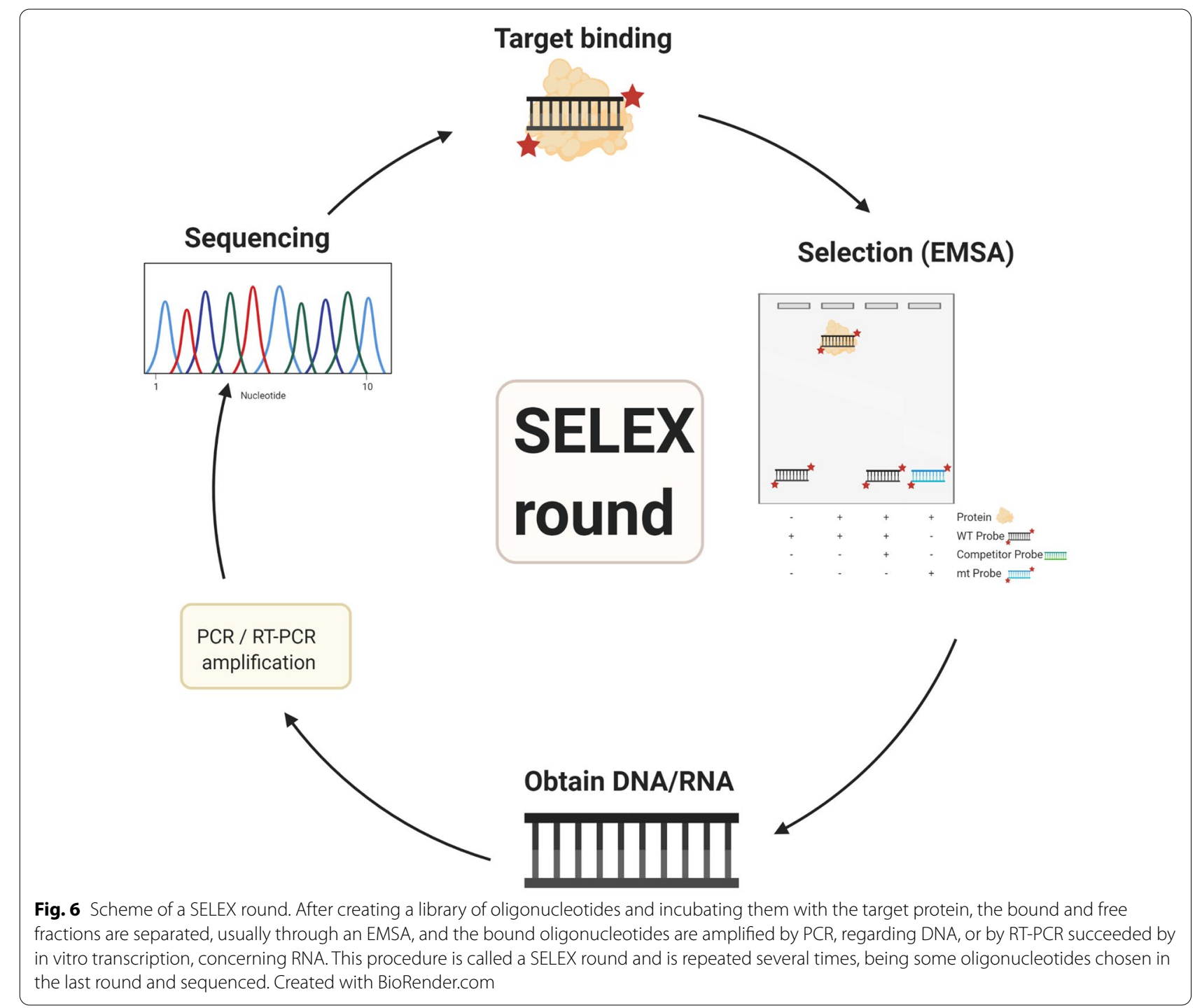

processes and other potential in vivo variables. This quite simple procedure identifies the ideal binding sequences for a given protein only requiring a relatively pure target protein sample, a method like EMSA to separate the bound and free fractions and a PCR to amplify the selected oligonucleotides. Moreover, the oligonucleotides used can be single or double-stranded DNA or RNA [52]. However, this technique also presents a drawback: several DNA-TF interactions have a low level of specificity and sensitivity. Prediction of the binding sites becomes more difficult when dealing with TFs that bind to several genes, presenting binding motifs with low information content [53]. Nevertheless, Roulet et al. [54] developed a high-throughput genomic method that fused SELEX with serial analysis of gene expression (SAGE) and an automated procedure to extract quality-controlled sequences, providing binding-site models with a higher accuracy and identifying additional regulatory sequences in genomic DNA. The advantages, disadvantages and applications of each one of these approaches are described in Table 2.

\section{DNA-protein binding quantification Filter binding assay and EMSA}

Apart from identifying nucleic acid-protein complexes and characterising these proteins, the filter binding assay and EMSA can also be useful to distinguish the complexes formed according to the equilibrium constants of each binding reaction, enabling to compare relative binding affinities and discriminate the interaction between a given protein with more than one nucleic acid sequence. To do so, it is essential to use a purified protein and know the respective concentration. Then, the affinity of a protein to a certain nucleic acid can be quantified performing a titration of the protein under study with a known 
concentration into a permanent concentration of the nucleic acid of interest [3].

Usually, the Michaelis-Menten analysis can be applied to examine these titrations, considering the protein added as the substrate, since the nucleic acid concentration is very low. This demands a high protein concentration to obtain a significant interaction, being the free and total protein concentrations quite similar. Thus, the equilibrium constant can be estimated as the concentration of protein necessary to retain, as for the filter binding assay [15], or shift the mobility, regarding the EMSA [17], of $50 \%$ of the nucleic acid. Furthermore, the increase in the amount of protein is accompanied by an increase in the amount of complex formed, until reaching a plateau, that corresponds to a saturation level, quite like the Michaelis-Menten curve [56]. Nevertheless, this analysis assumes a 1:1 stoichiometry and that all the protein is active [3].

Analysing DNA-protein interactions by intrinsic fluorescence, Carpenter et al. [57] developed a model according to which the use of high nucleic acid concentrations leads to a binding linear to the protein concentration $(P)$ until all the DNA is bound. At this point, the ratio between the protein and DNA concentrations gives the stoichiometry. If it is higher than 1 , it can mean that more than one protein link to the DNA, or that part of the protein is not active. Moreover, taking into consideration that, at the same moment, the concentration of the complex formed $(P D)$ is equal to the DNA concentration $(D)$ and that the equilibrium constant $(K)$ is calculated using the formula:

$$
K=\frac{P D}{P \times D},
$$

at this point $\mathrm{K}$ is equal to $\frac{1}{\mathrm{P}}$ [3]. However, in order to determine a high affinity, the protein concentration needs to be very low, which is difficult to measure. This way, in these cases, one can perform serial dilutions and calculate $P$ by the formula:

$$
P=P_{\text {total }}-P D,
$$

being the total concentration of protein $\left(P_{\text {total }}\right)$ calculated for each dilution and the $P D$ directly measured [58].

Following the same model, for intermediate DNA concentrations, when the stoichiometric point is reached, only a part of the DNA $(f)$ is linked to the protein $(P)$. Here the equilibrium constant $(K)$ is easily and accurately calculated by the formula:

$$
K=P \times \frac{(1-f)^{2}}{f} .
$$

However, unlike the previous case, here the DNA concentration needs to be acquired $[3,57]$.

Finally, competition assays can also be performed and equilibrium constants can be compared. In a first approach, the relative binding constant $\left(K_{r}\right)$ can be calculated using the formula: $K_{r}=\frac{K}{K_{c}}$, being $K_{c}$ the equilibrium constant of the reaction where the competing nucleic acid binds to the protein. This formula also represents the equilibrium constant of the overall reaction $\left(K_{T}\right)$. Taylor et al. [59] calculated the same overall equilibrium constant using the concentration $(C)$ of the competitor sequence needed to dissociate half of the specific complex and the formula.

$$
K_{T}=2 \times \frac{C}{D}-1 .
$$

Nevertheless, automated systems needed to be created. Initially developed by Gassmann et al. [60] and adapted for the study of DNA-protein interaction by Xian et al. [61], capillary electrophoresis-laser-induced fluorescence (CE-LIF) consists in submitting a DNA-protein complex to a capillary EMSA, using a laser-induced fluorescence detection system. It allows instant on-column visualisation, automated operation and computerised data analysis, enabling DNA-protein complex and DNA quantification and, consequently, stoichiometry determination. Moreover, it has small sample requirements, is highly sensitive and presents rapid analysis times [62]. Furthermore, combining CE with laser-induced fluorescence polarisation enables simultaneous measurements of electrophoretic mobility and fluorescence anisotropy ("Binding-site selection" section) [63]. Table 3 presents the advantages, disadvantages and applications of each one of these methods.

\section{Surface plasmon resonance}

In the beginning of the twentieth century, Wood [64] observed a dark and light bands pattern in the light reflected from a metal-backed diffraction grating exposed to polarised light. This phenomenon was explained later by Fano [65] as being related to surface waves, surface plasmon, supported by the grating. More than two decades after this theoretical analysis, Otto [66] demonstrated optical excitation of surface plasmons through attenuated total reflection. Thus, two ways of optically excite surface plasma waves are: attenuated total reflection in prism coupler-based structures and diffraction at gratings. Liedberg et al. [67] were pioneers in applying the first approach in sensing chemical substances, introducing the surface plasmon resonance (SPR) sensing technique, while Cullen et al. [68] were the first ones to make use of the second method for the same purpose. In the beginning of the 90's, the field 
Table 3 Advantages and disadvantages of techniques that describe DNA-binding reactions

\begin{tabular}{|c|c|c|c|c|}
\hline \multirow[t]{2}{*}{ Technique [References] } & \multicolumn{2}{|l|}{ Technique } & \multirow[t]{2}{*}{ Applications } & \multirow[t]{2}{*}{ Case studies } \\
\hline & Pros & Cons & & \\
\hline $\begin{array}{l}\text { Filter binding assay and } \\
\text { EMSA }[3,20,62]\end{array}$ & $\begin{array}{l}\text { The Michaelis-Menten analysis can } \\
\text { be applied use of intermediate DNA } \\
\text { concentrations enables to determine } \\
\text { directly and accurately the equilib- } \\
\text { rium constant } \\
\text { The use of high DNA concentrations } \\
\text { enables to determine the stoichi- } \\
\text { ometry } \\
\text { CE-LIF enables instant on-column } \\
\text { visualisation, automated operation } \\
\text { and computerised data analysis } \\
\text { CE-LIF has small sample requirements, } \\
\text { is highly sensitive and presents rapid } \\
\text { analysis times }\end{array}$ & $\begin{array}{l}\text { Need to use purified } \\
\text { protein and quantify } \\
\text { its concentration } \\
\text { The use of low DNA con- } \\
\text { centrations assumes } \\
\text { 1:1 stoichiometry } \\
\text { Need to perform serial } \\
\text { dilutions to determine } \\
\text { high affinities }\end{array}$ & $\begin{array}{l}\text { Compare binding affinities } \\
\text { CE-LIF enables DNA-protein complex } \\
\text { and DNA quantification and stoichi- } \\
\text { ometry determination } \\
\text { CE-LIF enables simultaneous measure- } \\
\text { ments of electrophoretic mobility } \\
\text { and fluorescence anisotropy }\end{array}$ & Prabu et al. [45] \\
\hline SPR [42] & $\begin{array}{l}\text { Very sensitive, fast and easy } \\
\text { Real-time assay } \\
\text { More adequate than EMSA when } \\
\text { comparing wild-type and mutant } \\
\text { proteins }\end{array}$ & $\begin{array}{l}\text { If more than one protein } \\
\text { bind cooperatively, } \\
\text { the results can be } \\
\text { misleading }\end{array}$ & $\begin{array}{l}\text { Measure binding affinities and kinetics } \\
\text { directly and simultaneously }\end{array}$ & Song et al. [74] \\
\hline
\end{tabular}

of bioanalysis with surface plasmon resonance had a huge development with the creation of SPR-biosensors machines, which apply the attenuated total reflection approach and include a dextran layer on the surface of a thin gold film, a laser beam and a diode array for detection [69]. Kinetic analysis of binding reactions [70] and DNA-protein interactions studies [71] that used these biosensors followed.

Basically, the SPR protocol for measuring biomolecular interactions starts by arresting one of the binding partners to the surface of a chip, followed by injecting the other binding partner. A real-time interaction curve is then recorded, measuring the increase in mass due to the binding as a function of time (Fig. 7). Flowing buffer over the chip leads to the dissociation of both partners and the signal decreases. This way, the kinetics of the interaction can be studied and the association and dissociation rate constants can be accessed [3].

Although, if more than one protein binds cooperatively, the results obtained with the SPR technique can be misleading and need a more complex analysis [3], SPR presents several advantages. It is a very sensitive method that directly measures binding affinities and kinetics simultaneously. Furthermore, this technique does not require labelling of the binding partners and is a real-time assay. Relatively to EMSA, SPR is faster, easier and more adequate when comparing wild-type and mutant proteins [42]. Even though, the immobilisation of the ligand in the SPR assay may affect its activity [72], Khan et al. [73] developed a new SPR approach where the protein binds to the chip at physiological conditions, which, relatively to standard immobilisation techniques, is fast, efficient and reversible. The advantages, disadvantages and applications of SPR are present in Table 3.

\section{Genome-wide techniques: ChIP and respective variants}

DNA fragments characteristics and sequences alone cannot be used to predict the genomic locations of bound proteins in a particular cell type. Thus, functional genome-wide approaches needed to be developed [75].

A few years after the first EMSA publication, Gilmour and Lis [76] created the Chromatin ImmunoPrecipitation (ChIP) technique, which revolutionised the study of DNA-protein interactions. This assay starts by crosslinking DNA and proteins through a living cells treatment with chemical cross-linkers [42] or UV light [76]. Then, cross-linked chromatin is extracted and fragmented by digestion or sonication. Finally, the DNA-protein complexes of interest are precipitated, usually using a specific antibody to the protein under study, and the immunoprecipitated DNA is purified, released from the cross-link and analysed by different methods according to the purpose of the experiment, such as Southern blotting, PCR, qPCR, hybridisation to arrays or cloning, and sequencing [42] (Fig. 8). Some of the methods that complement ChIP are discussed below.

Blat and Kleckner [77] broadened the range of the ChIP technique and developed the ChIP-chip assay, which combines ChIP with microarray technology. In this method, after reverting the cross-link, the immunoprecipitated DNA is labelled and hybridised to a microarray that contains a group of DNA sequences of interest. The same process is performed with a DNA sample that was 


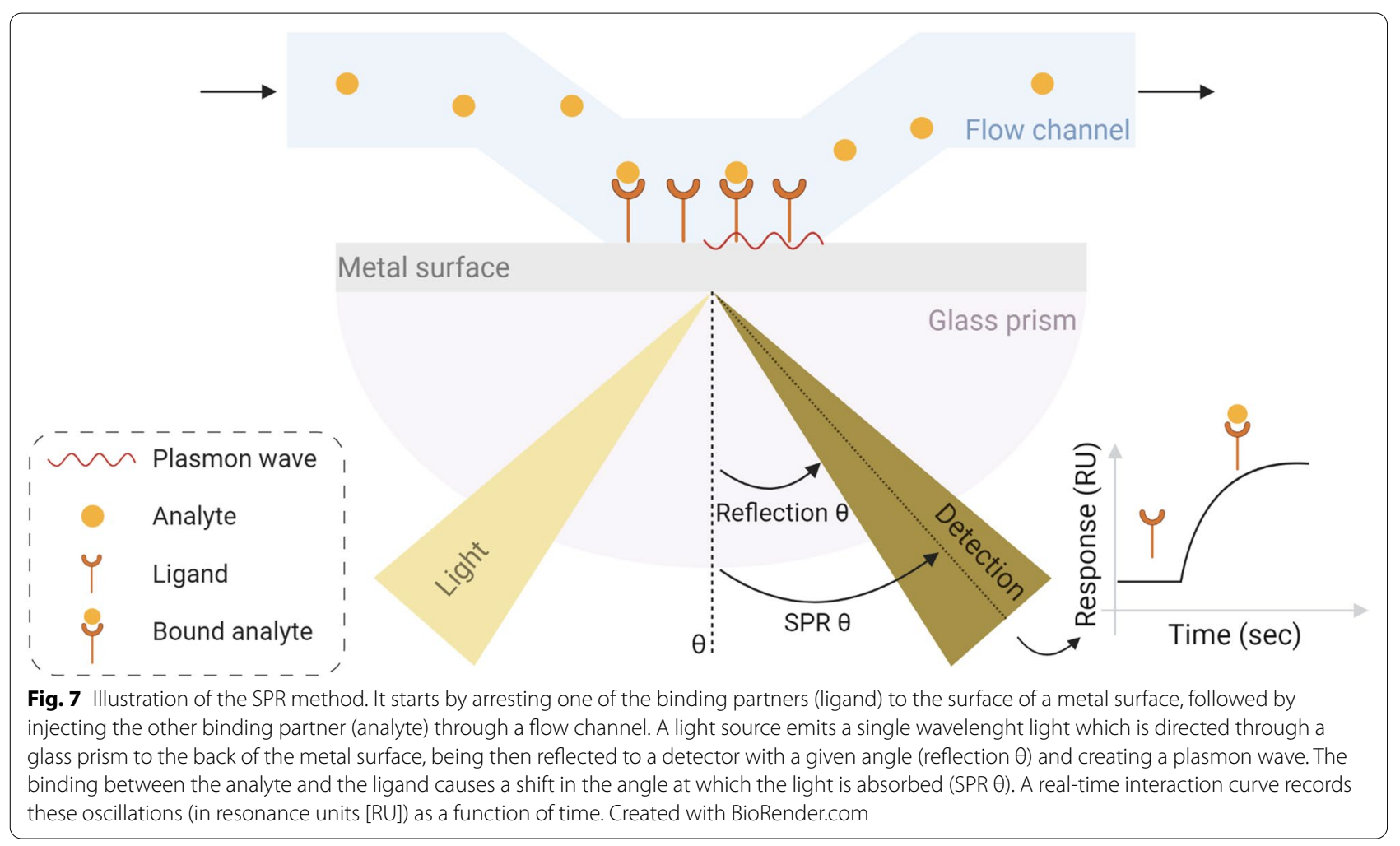

not precipitated as a control for variations in hybridisation intensity unrelated to the ChIP enrichment. Then, the relative binding of the protein under study to each sequence is calculated. While Blat and Kleckner [77] were investigating binding sites along yeast chromosome III, Ren et al. [78] performed a genome-wide study using a microarray that included all yeast intergenic sequences. Furthermore, the authors also combined both enriched and control DNA in the same microarray, but with different labels. Still, ChIP-chip presents several disadvantages. Firstly, amplification and hybridisation to probes of the immunoprecipitated fragments can introduce hybridisation noise signals from biased amplification. Also, the existence of different array designs and genome assemblies can lead to difficulties in comparing results from different groups.

In 2004, Impey et al. [79] developed a method combining ChIP with SAGE, which can be applied to genome scanning for TF binding site, and creating the ChIPSAGE assay. According to this technique, after obtaining the DNA fragments immunoprecipitated during ChIP and performing several amplification and restriction reactions, ditags are joined in order to create a concatemer that is transformed into bacteria, amplified through replication and isolated. Finally, each $21 \mathrm{bp}$ tag is sequenced and the respective recurrence is quantified. This technique comprehends the whole genome and does not require a priori acquaintance of sequences. Furthermore, the results obtained with these technique are measured more quantitatively, unlike ChIP-chip, that analyses spot intensities [79]. Nevertheless, this method involves several DNA amplification steps, which can introduce amplification bias. Moreover, although this technique helps in localising unique sites in the genome, it fails due to mapping ambiguity. Thus, ChIP-SAGE has a lower accuracy than ChIP-chip [80].

Two years later, Wei et al. [81] created a procedure quite similar to the previous one, where the immunoprecipitated fragments are cloned into a DNA library for propagation in bacteria. Then, the fragments are converted into paired-end ditags (PET), which are concatenated and cloned into a final plasmid for sequencing. These PET sequences are then mapped to the genome under study, with the possibility of originating a PET cluster when PETs from the same locus that contains the target binding site overlap [81]. This can lead to the prediction of new binding motifs present in the overlapping regions. Furthermore, this procedure does not introduce amplification bias. Thus, it improves the mapping accuracy of short-tags and increases the information content, relatively to the previous method. However, it also demands a large sequencing capacity [80].

Finally, the dawn of next generation sequencing (NGS) made it possible to decipher millions of DNA fragments 


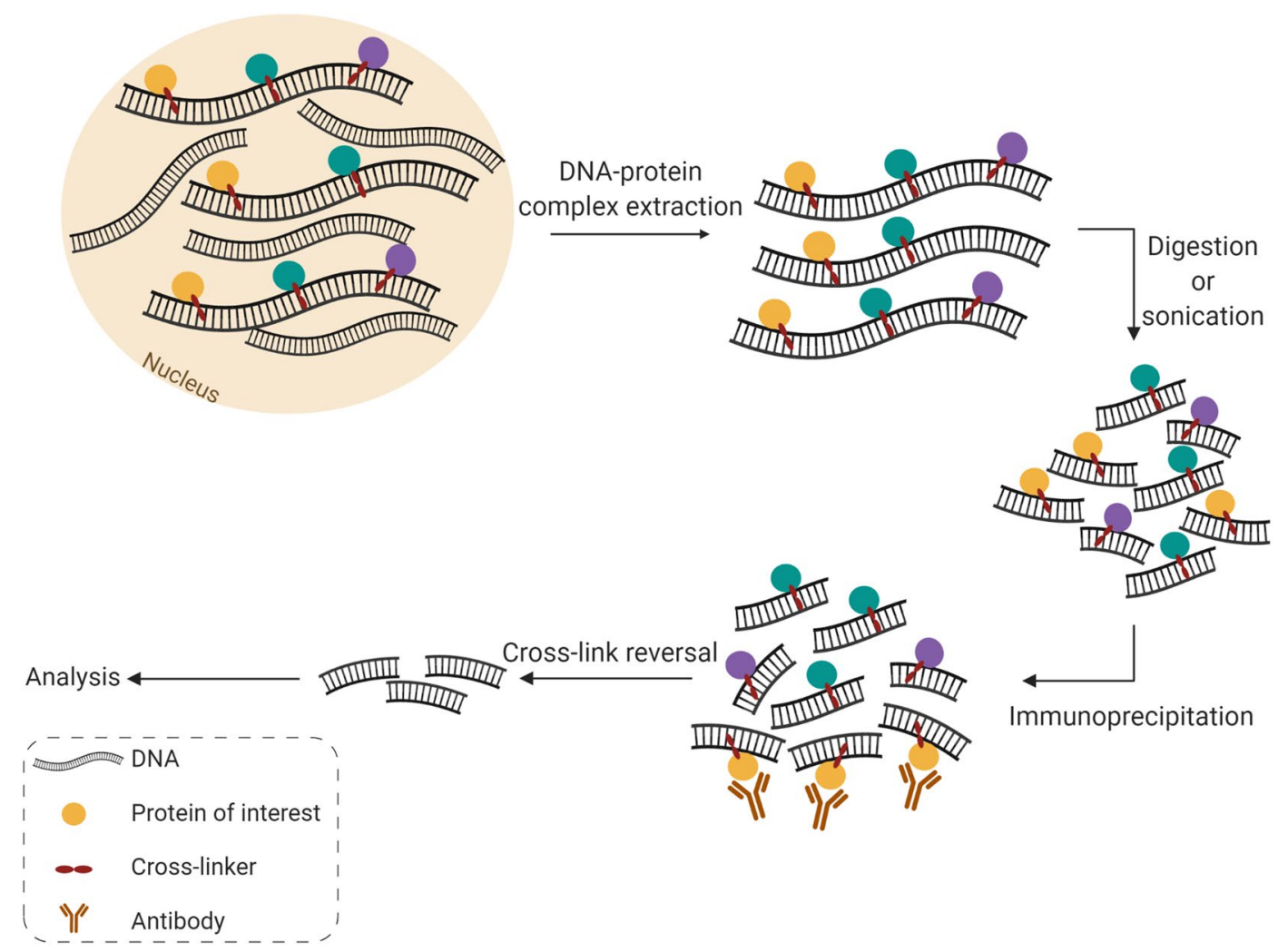

Fig. 8 Illustration of the ChIP procedure. ChIP techniques usually start by the cross-link between DNA and proteins in a living cells using chemical cross-linkers or UV light. Subsquently, cross-linked chromatin is extracted and fragmented by digestion or sonication. The DNA-protein complexes under study are then immunoprecipitated using a specific antibody to the protein of interest and the obtained DNA is purified, released from the cross-link and analysed. Created with BioRender.com

quickly, simultaneously and efficiently. In 2007, Johnson et al. [82] combined ChIP with ultrahigh-throughput DNA sequencing, creating a simple and robust technique for global, unbiased examination of the binding sites, the ChIP-seq. In this procedure, after making the ChIP DNA's ends blunt and ligating them with sequencing adaptors, limited PCR amplifications are performed. DNA fragments with selected sizes are amplified and sequenced in clusters using NGS technology. The sequences are then mapped to the genome of interest and regions with a high number of clusters of tag sequences are identified as ChIP enrichment sites. The non-specific sites are recognised, comparing the results with the ones obtained using a control DNA sequence, and removed [80].

In addition to the advantages of the previous two techniques, like the inexistence of hybridisation noise signals, the obtention of rigorous and quantifiable results and the genome coverage, the lower required amounts of ChIP DNA and, mainly, the higher base pair resolution are possibly the most notorious features of ChIP-seq. However, ChIP-seq also presents some disadvantages.
The choice of the antibody used is crucial in ChIP-seq, as well as in all ChIP techniques. Even extremely specific antibodies may react positively with nuclear proteins different from the one under study [50]. Furthermore, some sequencing errors can still exist, though the improvements that have been made to the NGS methods. Moreover, insufficient reads can lead to a loss of sensitivity and specificity in spotting the enriched regions. A large amount of sequencing may be needed in order to acquire a sufficient number of tags throughout the genome and obtain accurate estimations. Nevertheless, the dominant drawback of ChIP-seq is its high cost. Still, as the cost of sequencing continues to decrease, ChIP-seq is expected to become the desired method concerning ChIP experiments in the near future [83].

Another problem associated with ChIP-seq is its precision, since the DNA molecules present in the libraries that result from standard ChIP-seq experiments have a length of approximately $200 \mathrm{bp}$. However, a protein usually binds only $6-20$ bases. Furthermore, DNA contaminations are usual in these libraries, resulting from DNA that was not bound by the target protein and leading to 
systematic errors. In 2011, Rhee and Pugh [84] developed a new technique that eliminates this problem, ChIP-exo, according to which exonucleases digest free DNA fragments and link them to a fixed distance from the bound protein [75].

Other ChIP experiments have also been created, like Re-ChIP [85], according to which the binding to multiple proteins is tested for a single DNA sequence, and chromatin interaction studies [75]. The advantages, disadvantages and applications of each ChIP technique are present in Table 4.

\section{Case studies: recent uses and developments of techniques describing DNA-protein interactions}

Although some of the referred techniques are not so used currently, many of them were applied recently, sometimes complementing more modern techniques, or with slight modifications and improvements, proving their significance to unravel some aspects of the nucleic acidprotein interaction.

For instance, not long ago, Prabu et al. [45] combined the filter binding assay with SELEX in order to identify potent aptamers that bind to the Human Pituitary Tumour Transforming Gene 1 protein (PTTG1). Prabu et al. [45] were able to detect 3 aptamers that showed high frequencies of appearance. The authors also used the filter binding assay to determine the equilibrium dissociation constant of each aptamer, assuming a 1:1 binding stoichiometry between the aptamer and protein, and concluded that the third aptamer presented the higher binding affinity towards the PTTG1 protein.

Trying to decipher how the phytochrome-interacting factor 4 (PIF4) negatively regulates the transcription of the production of anthocyanin pigment1 (PAP1) gene in Arabidopsis thaliana seedlings, Liu et al. [46] performed a $\mathrm{Y} 1 \mathrm{H}$ to discover whether PIF4 binds to the promoter of $P A P 1$. The authors integrated PAP1 promoter sequences fused to a resistant gene into $\mathrm{Y} 1 \mathrm{H}$ Gold chromatin and identified three promoter sequences that interacted with PIF4, since the yeast strains generated presented high growth rates in media containing antibiotic. Furthermore, the authors also performed a ChIP-qPCR assay in order to test this interaction on Arabidopsis. After creating transgenic plants for the 35S:PIF4-HA construct and using anti-HA antibody in the ChIP-qPCR assay, Liu et al. [46] concluded that PIF4 had a higher affinity for the PAP1 binding site than the internal control (Actin2), suggesting that this factor could indeed bind to PAP1 in vivo. Moreover, the authors also performed an EMSA using the binding protein and a sequence containing the binding site. Liu et al. [46] concluded that PIF4 binds to the PAP1 gene promoter via the binding motif, since the electrophoretic lane containing the promoter sequence and PIF4 presented a delayed band, analogously to the lane containing the same parts together with a mutated competitor sequence and oppositely to what happened when using a mutated target sequence or when adding a wild-type competitor fragment. Finally, the authors also

Table 4 Advantages and disadvantages of ChIP techniques

\begin{tabular}{|c|c|c|c|c|}
\hline \multirow[t]{2}{*}{ Technique [References] } & \multicolumn{2}{|l|}{ Technique } & \multirow[t]{2}{*}{ Applications } & \multirow[t]{2}{*}{ Case studies } \\
\hline & Pros & Cons & & \\
\hline ChIP [42] & $\begin{array}{l}\text { In vivo method } \\
\text { Enables observation of highly } \\
\text { dynamic events }\end{array}$ & $\begin{array}{l}\text { Requires a population of cells } \\
\text { Cannot directly indicate functional } \\
\text { significance }\end{array}$ & $\begin{array}{l}\text { Predict the location of a bound } \\
\text { protein in a particular cell type }\end{array}$ & Liu et al. [46] \\
\hline ChIP-chip $[42,80]$ & $\begin{array}{l}\text { High throughput } \\
\text { Enables to calculate the relative } \\
\text { binding affinity of the protein } \\
\text { under study to each sequence }\end{array}$ & $\begin{array}{l}\text { Hybridisation noise signals from } \\
\text { biased amplification } \\
\text { Difficulties in comparing results } \\
\text { from different groups }\end{array}$ & $\begin{array}{l}\text { Detect the presence of a specific } \\
\text { protein throughout a large por- } \\
\text { tion of the genome }\end{array}$ & \\
\hline ChIP-SAGE [80] & $\begin{array}{l}\text { The results obtained are measured } \\
\text { more quantitatively }\end{array}$ & It suffers from mapping ambiguity & $\begin{array}{l}\text { Predict the location of a specific } \\
\text { protein throughout a large por- }\end{array}$ & \\
\hline ChIP-PET [80] & $\begin{array}{l}\text { The results obtained are measured } \\
\text { more quantitatively } \\
\text { Improves the mapping accuracy of } \\
\text { short-tags and the information } \\
\text { content }\end{array}$ & $\begin{array}{l}\text { Demands a large sequencing } \\
\text { capacity }\end{array}$ & tion or the entire genome & \\
\hline ChIP-seq $[75,80,83]$ & $\begin{array}{l}\text { Inexistence of hybridisation noise } \\
\text { signals } \\
\text { Obtention of rigorous and quanti- } \\
\text { fiable results }\end{array}$ & $\begin{array}{l}\text { Insufficient reads can lead to a loss } \\
\text { of sensitivity and specificity in } \\
\text { spotting the enriched regions } \\
\text { High cost }\end{array}$ & & \\
\hline ChIP-exo [75] & $\begin{array}{l}\text { Enables observation of highly } \\
\text { dynamic events }\end{array}$ & & $\begin{array}{l}\text { Predict the location of more than } \\
\text { one bound protein in a particu- } \\
\text { lar cell type }\end{array}$ & \\
\hline
\end{tabular}


performed a transient transcriptional expression analysis using Arabidopsis protoplasts, in order to understand if the interaction between the two parts would lead to the down-regulation of PAP1 expression. After introducing constructs containing the luciferase gene regulated by wild-type or mutated $P A P 1$ promoter sequences into different protoplasts together with constructs containing the PIF4 gene, Liu et al. [46] observed that the activation of the reporter gene was much lower in protoplasts containing constructs with the wild-type promoter and the PIF4 gene than in protoplasts with constructs containing the mutated promoter and the PIF4 gene. Thus, the authors concluded that PIF4 has a negative regulatory effect on the $P A P 1$ promoter function.

In order to identify the proteins that link to the Solanum lycopersicum LeSPL-CNR (squamosa promoter binding protein-like-colourless non-ripening) gene, Wang et al. [47] conducted a South-Western Blotting using a digoxigenin (DIG)-labelled 286 bp probe of this gene and a protein extract from tomato fruits. After performing a two-dimensional electrophoresis, the authors transferred the proteins to a PVDF membrane containing the labelled probes and identified 13 tomato proteins with known or predicted functions that linked to the fragment tested.

Recently, Manosas et al. [55] developed a high-throughput mechanism that can be applied to single molecule footprinting of small and large DNA ligands. Single molecule footprinting consists in applying a mechanical force with magnetic tweezers at the opposite ends of a DNA hairpin in order to disrupt the base-pairs, unzipping the DNA cooperatively in one step. However, the addition of ligands, such as proteins, leads to a multi-step untangle of the DNA caused by the ligand binding, enabling to determine the ligand sequence specificity. Through the measurement of the binding lifetimes at different forces, binding kinetics can be studied. The authors designed hairpin DNA molecules that allow to perform these measurements with a flat free energy landscape and test several binding sequences in a single assay, widening the repertoire of DNA footprinting assays.

Song et al. [74] used a biosensor surface in order to kinetically analyse the DNA-protein interactions of wildtype/mutant $\mathrm{p} 53$ proteins through real-time monitoring of the localised surface plasmon resonance shift. These case studies are referred in Tables 1, 2, 3 and 4.

\section{Conclusion}

Even though there are several and different techniques to describe, analyse and compare DNA-protein interactions, they can be organised into separate sections, according to the respective purpose. Filter binding assay and EMSA are easy in vitro methods that rapidly identify nucleic acid-protein binding interactions, being the procedures of choice in many functional studies that aim to confirm the gene targets of a given TF. Together with SDS-PAGE, EMSA can help in the identification of the proteins that bind to a certain sequence when using crude protein extracts. Cross-linking can also be used with this objective, especially when dealing with DNAprotein complexes that involve more than one protein that can be indirectly linked to the DNA. In vivo methods, like $\mathrm{Y} 1 \mathrm{H}$ and PTA, can also be applied when analysing if a TF binds to a given sequence. These approaches provide reliable results since they recreate similar environments to the ones where the complex is formed. When trying to discover the DNA-binding sites localisation, DNA-footprinting is indeed an easier, faster and relatively reliable approach, which also allows to investigate the impact of each nucleotide on the binding affinity. Furthermore, in vivo footprinting methods are also available. Nevertheless, techniques involving base analogues and base-site selection are more expensive, but confer a higher precision and are quite helpful when trying to identify aptamers that link to a given protein, as in the example described in the previous section. Quantifying the kinetics and affinity of a given DNA-protein interaction can be useful, mainly when comparing more than one interaction. Once again, filter binding assay and EMSA are useful and easy methods in this subject. SPR and methods applied to the proteins study that can also be used in the analysis of nucleic acid-protein interactions, like circular dichroism and fluorescence spectroscopy, are quite sensitive and direct approaches. However, these procedures involve specific instruments and technology. Finally, considering genome-wide studies, ChIP-seq is the desired method, given the coverage and resolution of the technique. Nevertheless, the costs related to NGS remain a barrier to the use of this technique. In conclusion, although some experiments are easier, less expensive and faster than others, when envisioning a DNA-protein interaction study several aspects cannot be disregarded, since several useful and available methods can confer more precise and accurate results in a simpler way than one may think.

\footnotetext{
Abbreviations

AD: Activation domain; CAP: Catabolite gene activator protein; CE-LIF: Capillary electrophoresis-laser-induced fluorescence; ChIP: Chromatin immunoprecipitation; DIG: Digoxigenin; EMSA: Electrophoretic mobility shift assay; LeSPL-CNR: Solanum lycopersicum squamosa promoter binding protein-likecolourless non-ripening; NGS: Next generation sequencing; PAP1: Production of anthocyanin pigment1; PET: Paired-end ditags; PIF4: Phytochrome-interacting factor 4; PTA: Protoplast transactivation assay; PTTG1: Human pituitary tumour transforming gene 1 protein; PVDF: Polyvinylidene difluoride; RU: Resonance units; SAGE: Serial analysis of gene expression; SDS-PAGE: Sodium dodecyl sulphate-polyacrylamide gel electrophoresis; SELEX: Systematic evolution of ligands by exponential enrichment; SPR: Surface plasmon resonance; TEAMP: Transient expression in arabidopsis mesophyll protoplast; TF: Transcription factor; WOX8: Wuschel related homeobox 8; Y1 H: Yeast one-hybrid.
} 


\section{Acknowledgements}

The work described in this review was supported by the EU project 690946-SexSeed-Sexual Plant Reproduction-Seed Formation, funded by H2020-MSCA-RISE-2015, and SeedWheels FCT Project-POCI-01-0145FEDER-027839; ALL's research was supported by an FCT PhD grant SFRH/ BD/115960/2016; JS has received funding from "la Caixa" Foundation (ID 100010434), under the agreement LCF/BQ/DR20/11790010; DM's research was supported by an FCT PhD grant SFRH/BD/143557/2019; MJF's research was supported by an FCT PhD grant SFRH/BD/143579/2019.

\section{Authors' contributions}

RF organised and wrote the manuscript. ALL assisted in writing and revising the manuscript. JS assisted in writing the manuscript. DM and MJF organised and prepared the figures. SC proposed and corrected the manuscript. All authors read and approved the final manuscript.

\section{Funding}

The work described in this review was supported by the EU project 690946-SexSeed-Sexual Plant Reproduction-Seed Formation, funded by H2020-MSCA-RISE-2015, and SeedWheels FCT Project-POCl-01-0145-FEDER-027839.

\section{Availability of data and materials}

Not applicable.

\section{Declarations}

\section{Ethics approval and consent to participate}

Not applicable.

\section{Consent for publication}

Not applicable.

\section{Competing interests}

The authors declare that they have no competing interests.

\section{Author details}

${ }^{1}$ Departamento de Biologia, Faculdade de Ciências da Universidade do Porto, Porto, Portugal. ${ }^{2}$ LAQV Requimte, Sustainable Chemistry, Universidade do Porto, Porto, Portugal. ${ }^{3}$ Universidade do Minho, Braga, Portugal.

Received: 21 January 2021 Accepted: 11 July 2021

Published online: 23 July 2021

\section{References}

1. Stedman E, Stedman E. Cell specificity of histones. Nature. 1950;166(4227):780-1.

2. Ofran Y, Mysore $V$, Rost B. Prediction of DNA-binding residues from sequence. Bioinformatics. 2007;23(13):i347-53.

3. Guille MJ, Kneale GG. Methods for the analysis of DNA-protein interactions. Mol Biotechnol. 1997;8(1):35-52

4. Leng M, Felsenfe $G$. Preferential interactions of polylysine and polyarginine with specific base sequences in DNA. Proc Natl Acad Sci USA. 1966;56(4):1325.

5. Seeman NC, Rosenberg JM, Rich A. Sequence-specific recognition of double helical nucleic-acids by proteins. Proc Natl Acad Sci USA. 1976;73(3):804-8.

6. McKay DB, Steitz TA. Structure of catabolite gene activator protein at 2.9 A resolution suggests binding to left-handed B-DNA. Nature. 1981;290(5809):744-9.

7. Pabo CO, Sauer RT. Protein-DNA recognition. Annu Rev Biochem. 1984;53(1):293-321.

8. Matthews BW. No code for recognition. Nature. 1988;335(6188):294-5

9. Luscombe NM, Laskowski RA, Thornton JM. Amino acid-base interactions: a three-dimensional analysis of protein-DNA interactions at an atomic level. Nucleic Acids Res. 2001;29(13):2860-74.
10. Jacob F, Monod J. Genetic regulatory mechanisms in the synthesis of proteins. J Mol Biol. 1961;3(3):318-56.

11. Yarus M, Berg P. Recognition of Trna by Aminoacyl Trna Synthetases. J Mol Biol. 1967;28(3):479-80.

12. Riggs $A D$, Suzuki $H$, Bourgeois S. Lac repressor-operator interaction. 1. Equilibrium studies. J Mol Biol. 1970;48(1):67.

13. Nirenberg MW, Leder P. Rna codewords + protein synthesis-effect of trinucleotides upon binding of Srna to ribosomes. Science. 1964;145(363):1399-2000.

14. Jones OW, Berg P. Studies on binding of RNA polymerase to polynucleotides. J Mol Biol. 1966;22(2):199-200.

15. Riggs AD, Bourgeois $S$, Newby RF, Cohn M. DNA binding of the lac repressor. J Mol Biol. 1968;34(2):365-8.

16. Hall KB, Kranz JK. Nitrocellulose filter binding for determination of dissociation constants. Methods Mol Biol. 1999;118:105-14.

17. Fried M, Crothers DM. Equilibria and kinetics of lac repressor-operator interactions by polyacrylamide gel electrophoresis. Nucleic Acids Res. 1981;9(23):6505-25.

18. Hellman LM, Fried MG. Electrophoretic mobility shift assay (EMSA) for detecting protein-nucleic acid interactions. Nat Protoc. 2007;2(8):1849.

19. Garner MM, Revzin A. A gel electrophoresis method for quantifying the binding of proteins to specific DNA regions: application to components of the Escherichia coli lactose operon regulatory system. Nucleic Acids Res. 1981;9(13):3047-60.

20. Fried MG. Measurement of protein-DNA interaction parameters by electrophoresis mobility shift assay. Electrophoresis. 1989;10(5-6):366-76.

21. Das M, Fox CF. Chemical cross-linking in biology. Annu Rev Biophys Bioeng. 1979;8(1):165-93.

22. Welsh J, Cantor CR. Protein-DNA cross-linking. Trends Biochem Sci. 1984;9(12):505-8.

23. Brutlag D, Schlehuber C, Bonner J. Properties of formaldehyde-treated nucleohistone. Biochemistry. 1969;8(8):3214-8.

24. Chalkley R, Hunter C. Histone-histone propinquity by aldehyde fixation of chromatin. Proc Natl Acad Sci. 1975;72(4):1304-8.

25. Kuykendall JR, Bogdanffy MS. Efficiency of DNA-histone crosslinking induced by saturated and unsaturated aldehydes in vitro. Mutat Res Lett. 1992;283(2):131-6.

26. Chattoraj DK, Inman RB. Location of DNA ends in P2, 186, P4 and lambda bacteriophage heads. J Mol Biol. 1974;87(1):11-22.

27. Thomas $\mathrm{JO}$, Sternberg $\mathrm{N}$, Weisberg R. Altered arrangement of the DNA in injection-defective lambda bacteriophage. J Mol Biol. 1978;123(2):149-61.

28. Smith KC. Dose dependent decrease in extractability of DNA from bacteria following irradiation with ultraviolet light or with visible light plus dye. Biochem Biophys Res Commun. 1962;8(3):157-63.

29. Lica L, Ray DS. Replication of bacteriophage M13: XII. In vivo crosslinking of a phage-specific DNA binding protein to the single-stranded DNA of bacteriophage M13 by ultraviolet irradiation. J Mol Biol. 1977;115(1):45-59.

30. Cao TM, Sung MT. Ultraviolet light induced preferential crosslinking of histone $\mathrm{H} 3$ to DNA in chromatin and nuclei of chicken erythrocytes. Biochemistry. 1982;21(14):3419-27.

31. Harrison CA, Turner DH, Hinkle DC. Laser crosslinking of E. coli RNA polymerase and T7 DNA. Nucleic Acids Res. 1982;10(7):2399-414.

32. Towbin H, Staehelin T, Gordon J. Electrophoretic transfer of proteins from polyacrylamide gels to nitrocellulose sheets: procedure and some applications. Proc Natl Acad Sci. 1979;76(9):4350-4.

33. Kristie TM, Roizman B. Alpha 4, the major regulatory protein of herpes simplex virus type 1 , is stably and specifically associated with promoterregulatory domains of alpha genes and of selected other viral genes. Proc Natl Acad Sci. 1986;83(10):3218-22.

34. Buratowski S, Chodosh LA. Mobility shift DNA-binding assay using gel electrophoresis. Curr Protoc Pharmacol. 2001;13(1):6.8.1-6.8.12.

35. Singh $H$, LeBowitz $J$, Baldwin AS, Sharp PA. Molecular cloning of an enhancer binding protein: isolation by screening of an expression library with a recognition site DNA. Cell. 1988;52(3):415-23.

36. Demczuk S, Harbers M, Vennström B. Identification and analysis of all components of a gel retardation assay by combination with immunoblotting. Proc Natl Acad Sci. 1993;90(7):2574-8.

37. Granger-Schnarr M, Lloubes R, de Murcia G, Schnarr M. Specific proteinDNA complexes: immunodetection of the protein component after gel electrophoresis and Western blotting. Anal Biochem. 1988;174(1):235-8. 
38. Chen H, Chang GD. Simultaneous immunoblotting analysis with activity gel electrophoresis in a single polyacrylamide gel. Electrophoresis. 2001;22(10):1894-9.

39. Stead JA, Keen JN, McDowall KJ. The identification of nucleic acid-interacting proteins using a simple proteomics-based approach that directly incorporates the electrophoretic mobility shift assay. Mol Cell Proteomics. 2006;5(9):1697-702.

40. Li JJ, Herskowitz I. Isolation of ORC6, a component of the yeast origin recognition complex by a one-hybrid system. Science. 1993:262(5141):1870-4.

41. Reece-Hoyes JS, Walhout AM. Yeast one-hybrid assays: a historical and technical perspective. Methods. 2012;57(4):441-7.

42. Cai $\mathrm{YH}, \mathrm{Huang} \mathrm{H}$. Advances in the study of protein-DNA interaction. Amino Acids. 2012;43(3):1141-6.

43. Yoo S-D, Cho Y-H, Sheen J. Arabidopsis mesophyll protoplasts: a versatile cell system for transient gene expression analysis. Nat Protoc. 2007;2(7):1565.

44. Ueda M, Zhang Z, Laux T. Transcriptional activation of Arabidopsis axis patterning genes WOX8/9 links zygote polarity to embryo development. Dev Cell. 2011;20(2):264-70.

45. Prabu SS, Chng ES, Woon PY, Chen J-H, Tang T-H, Citartan M. Unravelling the diagnostic and therapeutic potentialities of a novel RNA aptamer isolated against human pituitary tumour transforming gene 1 (PTTG1) protein. Anal Chim Acta. 2020;1138:181-90.

46. Liu Z, Wang Y, Fan K, Li Z, Jia Q, Lin W, et al. PHYTOCHROME-INTERACTING FACTOR 4 (PIF4) negatively regulates anthocyanin accumulation by inhibiting PAP1 transcription in Arabidopsis seedlings. Plant Sci. 2020;303:110788.

47. Wang $X$, Ye B, Wang Y, Zhou T, Lai T. In search of regulators of LeSPL-CNR by south-western blotting and yeast one-hybrid library screening system. Am J Plant Sci. 2018;9(05):1037.

48. Papavassiliou AG, Heumann H. 1,10-Phenanthroline-copper ion nuclease footprinting of DNA-protein complexes in situ following mobility-shift electrophoresis assays. In: DNA-protein interactions. NewJersey: Springer; 1994. p. 43-78.

49. Church GM, Gilbert W. Genomic sequencing. Proc Natl Acad Sci. 1984;81(7):1991-5.

50. Emamjomeh A, Choobineh D, Hajieghrari B, MahdiNezhad N, Khodavirdipour A. DNA-protein interaction: identification, prediction and data analysis. Mol Biol Rep. 2019;46(3):3571-96.

51. Yansura D, Goeddel D, Cribbsand D, Caruthers M. Studies on gene control regions. III. Binding of synthetic and modified synthetic lac operator DNAs to lactose repressor. Nucleic Acids Res. 1977;4(3):723-37.

52. Tuerk C, Gold L. Systematic evolution of ligands by exponential enrichment: RNA ligands to bacteriophage T4 DNA polymerase. Science. 1990;249(4968):505-10.

53. Sengupta AM, Djordjevic M, Shraiman BI. Specificity and robustness in transcription control networks. Proc Natl Acad Sci. 2002;99(4):2072-7.

54. Roulet E, Busso S, Camargo AA, Simpson AJ, Mermod N, Bucher P. Highthroughput SELEX-SAGE method for quantitative modeling of transcription-factor binding sites. Nat Biotechnol. 2002;20(8):831-5.

55. Manosas M, Camunas-Soler J, Croquette V, Ritort F. Single molecule highthroughput footprinting of small and large DNA ligands. Nat Commun. 2017;8(1):1-12.

56. Taylor JD, Ackroyd AJ, Halford SE. The gel shift assay for the analysis of DNAprotein interactions. In: DNA-protein interactions. New Jersey: Springer; 1994. p. 263-79.

57. Carpenter ML, Oliver AW, Kneale GG. Analysis of DNA-protein interactions by intrinsic fluorescence. In: DNA-protein interactions. New Jersey: Springer; 2001. p. 491-502.

58. Fried MG, Crothers DM. Equilibrium studies of the cyclic AMP receptor protein-DNA interaction. J Mol Biol. 1984;172(3):241-62.

59. Taylor I, Watts D, Kneale G. Substrate recognition and selectivity in the type IC DNA modification methylase M. EcoR124I. Nucleic Acids Res. 1993;21(21):4929-35.

60. Gassmann E, Kuo J, Zare R. Electrokinetic separation of chiral compounds. Science. 1985;230(4727):813-4.

61. Xian J, Harrington MG, Davidson EH. DNA-protein binding assays from a single sea urchin egg: a high-sensitivity capillary electrophoresis method. Proc Natl Acad Sci. 1996;93(1):86-90.

62. Ronai Z, Wang Y, Khandurina J, Budworth P, Sasvari-Szekely M, Wang X, et al. Transcription factor binding study by capillary zone electrophoretic mobility shift assay. Electrophoresis. 2003;24(1-2):96-100.
63. Wan Q-H, Le XC. Studies of protein-DNA interactions by capillary electrophoresis/laser-induced fluorescence polarization. Anal Chem. 2000;72(22):5583-9.

64. Wood RWXLII. On a remarkable case of uneven distribution of light in a diffraction grating spectrum. London Edinburgh Dublin Philos Mag J Sci. 1902;4(21):396-402.

65. Fano $U$. The theory of anomalous diffraction gratings and of quasi-stationary waves on metallic surfaces (Sommerfeld's waves). JOSA. 1941;31(3):213-22.

66. Otto A. Excitation of nonradiative surface plasma waves in silver by the method of frustrated total reflection. Z Physik A Hadrons Nuclei. 1968;216(4):398-410.

67. Liedberg B, Nylander C, Lunström I. Surface plasmon resonance for gas detection and biosensing. Sens Actuators. 1983;4:299-304.

68. Cullen D, Brown R, Lowe C. Detection of immuno-complex formation via surface plasmon resonance on gold-coated diffraction gratings. Biosensors. 1987;3(4):211-25.

69. Löfås S, Malmqvist M, Rönnberg I, Stenberg E, Liedberg B, Lundström I. Bioanalysis with surface plasmon resonance. Sens Actuators B Chem. 1991;5(1-4):79-84.

70. Karlsson R, Michaelsson A, Mattsson L. Kinetic analysis of monoclonal antibody-antigen interactions with a new biosensor based analytical system. J Immunol Methods. 1991;145(1-2):229-40.

71. Bondeson K, Frostellkarlsson A, Fagerstam L, Magnusson G. Lactose repressor-operator DNA interactions: kinetic analysis by a surface plasmon resonance biosensor. Anal Biochem. 1993;214(1):245-51.

72. Ling J, Liao H, Clark R, Wong MSM, Lo DD. Structural constraints for the binding of short peptides to claudin-4 revealed by surface plasmon resonance. J Biol Chem. 2008;283(45):30585-95.

73. Khan SH, Farkas K, Kumar R, Ling J. A versatile method to measure the binding to basic proteins by surface plasmon resonance. Anal Biochem. 2012:421(2):385-90.

74. Song S, Lee JU, Kang J, Park KH, Sim SJ. Real-time monitoring of distinct binding kinetics of hot-spot mutant p53 protein in human cancer cells using an individual nanorod-based plasmonic biosensor. Sens Actuators B Chem. 2020;322:128584.

75. Furey TS. ChIP-seq and beyond: new and improved methodologies to detect and characterize protein-DNA interactions. Nat Rev Genet. 2012;13(12):840-52.

76. Gilmour DS, Lis JT. In vivo interactions of RNA polymerase II with genes of Drosophila melanogaster. Mol Cell Biol. 1985;5(8):2009-18.

77. Blat Y, Kleckner N. Cohesins bind to preferential sites along yeast chromosome III, with differential regulation along arms versus the centric region. Cell. 1999;98(2):249-59.

78. Ren B, Robert F, Wyrick JJ, Aparicio O, Jennings EG, Simon I, et al. Genome-wide location and function of DNA binding proteins. Science. 2000;290(5500):2306-9.

79. Impey S, McCorkle SR, Cha-Molstad H, Dwyer JM, Yochum GS, Boss JM, et al. Defining the CREB regulon: a genome-wide analysis of transcription factor regulatory regions. Cell. 2004;119(7):1041-54.

80. Wong E, Wei C-L. ChIP'ing the mammalian genome: technical advances and insights into functional elements. Genome Med. 2009;1(9):1-10.

81. Wei C-L, Wu Q, Vega VB, Chiu KP, Ng P, Zhang T, et al. A global map of p53 transcription-factor binding sites in the human genome. Cell. 2006;124(1):207-19.

82. Johnson DS, Mortazavi A, Myers RM, Wold B. Genome-wide mapping of in vivo protein-DNA interactions. Science. 2007;316(5830):1497-502.

83. Park PJ. ChIP-seq: advantages and challenges of a maturing technology. Nat Rev Genet. 2009;10(10):669-80.

84. Rhee HS, Pugh BF. Comprehensive genome-wide protein-DNA interactions detected at single-nucleotide resolution. Cell. 2011;147(6):1408-19.

85. Scully KM, Jacobson EM, Jepsen K, Lunyak V, Viadiu H, Carrière C, et al. Allosteric effects of Pit-1 DNA sites on long-term repression in cell type specification. Science. 2000;290(5494):1127-31.

\section{Publisher's Note}

Springer Nature remains neutral with regard to jurisdictional claims in published maps and institutional affiliations. 